\title{
A new type of CP symmetry, family replication and fermion mass hierarchies
}

\author{
M. Maniatis * * von Manteuffel * $^{\dagger}$ and O. Nachtmann \\ Institut für Theoretische Physik, Philosophenweg 16, 69120 Heidelberg, Germany
}

\begin{abstract}
We study a two-Higgs-doublet model with four generalised CP symmetries in the scalar sector. Electroweak symmetry breaking leads automatically to spontaneous breaking of two of them. We require that these four CP symmetries can be extended from the scalar sector to the full Lagrangian and call this requirement the principle of maximal CP invariance. The Yukawa interactions of the fermions are severely restricted by this requirement. In particular, a single fermion family cannot be coupled to the Higgs fields. For two fermion families, however, this is possible. Enforcing the absence of flavour-changing neutral currents, we find degenerate masses in both families or one family massless and one massive. In the latter case the Lagrangian is highly symmetric, with the mass hierarchy being generated by electroweak symmetry breaking. Adding a third family uncoupled to the Higgs fields and thus keeping it massless we get a model which gives a rough approximation of some features of the fermions observed in Nature. We discuss a number of predictions of the model which may be checked in future experiments at the LHC.
\end{abstract}

\section{INTRODUCTION}

In the Standard Model of particle physics (SM) we have three families of fermions, leptons and quarks, the electroweak gauge bosons $\gamma, W^{ \pm}, Z$, the gluon $G$ as the strong interaction gauge boson and one scalar Higgs doublet field [1, 2, 3]. For an introduction to the physics of the SM see for instance [4]. Our notations and kinematics conventions follow this reference. The SM gives, however, no explanation why there should be a replication of families in Nature. Also, the fermion masses, arising from the Yukawa interactions of the fermions with the Higgs field, remain arbitrary.

In this paper we present some considerations based on a two-Higgs-doublet model (THDM). We show that a certain type of CP symmetry, which is possible for a THDM forbids a non-zero Yukawa coupling to one fermion family only. However, an invariant coupling to two fermion families can be constructed. Moreover, this new type of CP symmetry is automatically spontaneously broken by the electroweak symmetry breaking (EWSB). As we shall show, this also leads to interesting results for the masses in the two fermion families. In essence we find that in this type of theories absence of large flavour-changing neutral currents (FCNCs) can only be achieved if the corresponding fermions in the two families have equal masses or if one family is massive, one massless.

Various aspects of two-Higgs-doublet models have been investigated in the literature; see for instance $[\underline{5}, \underline{6}, \mathbf{7}, \underline{8}$, 9, 10, 11, 12, 13, 14, 15] and references therein. In our group we have made a systematic study of the stability and symmetry breaking in the most general THDM [16]. In [17] we have made a systematic investigation of all possible types of generalised CP transformations for the two Higgs doublets. We have also given the criteria

\footnotetext{
*E-mail: M.Maniatis@thphys.uni-heidelberg.de

${ }^{\dagger}$ E-mail: A.v.Manteuffel@thphys.uni-heidelberg.de

${ }^{\ddagger}$ E-mail: O.Nachtmann@thphys.uni-heidelberg.de
}

for CP invariance of the two-Higgs-doublet potential and for spontaneous $\mathrm{CP}$ violation. In particular, we classified the generalised CP transformations as type (i) and type (ii). The structure of the Higgs sector of theories with type (i) CP invariance was discussed. In the present work we continue the investigation of THDMs with type (i) CP invariance in view of the possibilities for invariant fermion-Higgs-boson couplings in this framework. Indeed, the paper [17] and the present paper are companion papers. Therefore sections and equations of [17] will be quoted as section I.1 etc. and (I.1), (I.2) etc. respectively.

Our paper is organised as follows. In section 2 we recall the main results of [17] concerning THDMs with generalised CP invariance of type (i). In section 3 we discuss the vacuum expectation values (VEVs) and the physical Higgs mass spectrum of our models. In section 4 we introduce the fermion families and consider their coupling to the Higgs fields. In section 5 we collect our results and discuss their physical consequences. Section [6] contains our conclusions. Detailed derivations of various results are presented in the appendices.

\section{THE THDM WITH CP TYPE (i) INVARIANCE}

We consider models with the particle content as in the SM but with $n$ fermion families $(n=1,2,3)$ and with two Higgs doublet fields instead of one. The two Higgs fields are denoted by

$$
\varphi_{i}(x)=\left(\begin{array}{c}
\varphi_{i}^{+}(x) \\
\varphi_{i}^{0}(x)
\end{array}\right)
$$

with $i=1,2$. The gauge group of our models is assumed to be the usual one, $S U(3)_{C} \times S U(2)_{L} \times U(1)_{Y}$. In the following the gauge group $S U(3)_{C}$ of strong interactions will play no role. We shall be concerned with the gauge groups of weak isospin, $S U(2)_{L}$, and weak hypercharge $U(1)_{Y}$. Both Higgs doublets in (11) are assigned weak 
hypercharge $y=1 / 2$. The most general gauge-invariant Lagrange density can then be written as

$$
\mathscr{L}_{\mathrm{THDM}}=\mathscr{L}_{\varphi}+\mathscr{L}_{\mathrm{Yuk}}+\mathscr{L}_{\mathrm{FB}}
$$

Here $\mathscr{L}_{\mathrm{FB}}$ is the standard gauge kinetic Lagrange density for fermions and gauge bosons; see for instance [4]. The Higgs-boson Lagrangian is

$$
\mathscr{L}_{\varphi}=\sum_{i=1,2}\left(D_{\mu} \varphi_{i}\right)^{\dagger}\left(D^{\mu} \varphi_{i}\right)-V\left(\varphi_{1}, \varphi_{2}\right)
$$

with $V\left(\varphi_{1}, \varphi_{2}\right)$ the Higgs-boson potential. Finally, the Yukawa term, denoted by $\mathscr{L}_{\text {Yuk }}$, contains the Higgsboson-fermion couplings which will be the main focus of study in this paper.

Let us, however, first recall the main result from [16] and [17] concerning the Higgs potential $V$ and CP transformations in the Higgs sector. We use the framework of gauge-invariant functions. For this we introduce the $2 \times 2$ matrix of the Higgs fields (see (A.2) of [16] and (I.8)),

$$
\phi(x)=\left(\begin{array}{cc}
\varphi_{1}^{+}(x) & \varphi_{1}^{0}(x) \\
\varphi_{2}^{+}(x) & \varphi_{2}^{0}(x)
\end{array}\right) .
$$

Similarly, the scalar products of two Higgs fields, $\varphi_{i}^{\dagger}(x) \varphi_{j}(x)(i, j \in\{1,2\})$ are grouped into a $2 \times 2$ hermitian matrix

$$
\begin{aligned}
\underline{K}(x) & =\phi(x) \phi^{\dagger}(x) \\
& =\frac{1}{2}\left(K_{0}(x) \mathbb{1}_{2}+\mathbf{K}(x) \boldsymbol{\sigma}\right) .
\end{aligned}
$$

Here we have expanded $\underline{K}(x)$ in terms of the unit matrix $\mathbb{1}_{2}$ and the Pauli matrices $\sigma^{a}(a=1,2,3)$, thus defining the gauge-invariant functions

$$
\tilde{\mathbf{K}}(x)=\left(\begin{array}{c}
K_{0}(x) \\
\mathbf{K}(x)
\end{array}\right) .
$$

These form a real four-vector parametrising the gauge orbits of the Higgs fields. As we see immediately from (5) $\underline{K}(x)$ is a positive semidefinite $2 \times 2$ matrix. This implies that $\tilde{\mathbf{K}}(x)$ must be on or inside the forward light cone:

$$
\begin{aligned}
\tilde{\mathbf{K}}^{\mathrm{T}}(x) \tilde{g} \tilde{\mathbf{K}}(x) & \geq 0 \\
K_{0}(x) & \geq 0
\end{aligned}
$$

where

$$
\tilde{g}=\operatorname{diag}(1,-1,-1,-1) .
$$

The most general $S U(2)_{L} \times U(1)_{Y}$-invariant potential can now be written as

$$
\begin{aligned}
V= & \tilde{\boldsymbol{\xi}}^{\mathrm{T}} \tilde{\mathbf{K}}(x)+\tilde{\mathbf{K}}^{\mathrm{T}}(x) \tilde{E} \tilde{\mathbf{K}}(x) \\
= & \xi_{0} K_{0}(x)+\boldsymbol{\xi}^{\mathrm{T}} \mathbf{K}(x)+\eta_{00} K_{0}^{2}(x) \\
& +2 K_{0}(x) \boldsymbol{\eta}^{\mathrm{T}} \mathbf{K}(x)+\mathbf{K}^{\mathrm{T}}(x) E \mathbf{K}(x) .
\end{aligned}
$$

Here the potential parameters are a real four-vector and a real symmetric $4 \times 4$ matrix:

$$
\begin{gathered}
\tilde{\boldsymbol{\xi}}=\left(\begin{array}{c}
\xi_{0} \\
\boldsymbol{\xi}
\end{array}\right), \quad \tilde{E}=\left(\begin{array}{cc}
\eta_{00} & \boldsymbol{\eta}^{\mathrm{T}} \\
\boldsymbol{\eta} & E
\end{array}\right), \\
\boldsymbol{\xi}=\left(\begin{array}{l}
\xi_{1} \\
\xi_{2} \\
\xi_{3}
\end{array}\right), \quad \boldsymbol{\eta}=\left(\begin{array}{l}
\eta_{1} \\
\eta_{2} \\
\eta_{3}
\end{array}\right), \\
E=E^{\mathrm{T}}=\left(\begin{array}{lll}
\eta_{11} & \eta_{12} & \eta_{13} \\
\eta_{21} & \eta_{22} & \eta_{23} \\
\eta_{31} & \eta_{32} & \eta_{33}
\end{array}\right) .
\end{gathered}
$$

Under a basis change of the Higgs doublets

$$
\varphi_{i}^{\prime}(x)=U_{i j} \varphi_{j}(x), \quad i, j \in\{1,2\},
$$

with $U=\left(U_{i j}\right) \in U(2)$ the functions $K_{0}(x), \mathbf{K}(x)$ transform as

$$
\begin{aligned}
K_{0}^{\prime}(x) & =K_{0}(x), \\
\mathbf{K}^{\prime}(x) & =R(U) \mathbf{K}(x) .
\end{aligned}
$$

Here $R(U)$ is a proper rotation matrix $(R(U) \in S O(3))$ obtained from

$$
U^{\dagger} \sigma^{a} U=R_{a b}(U) \sigma^{b}, \quad a, b \in\{1,2,3\} .
$$

The potential (9) stays the same under such a basis change if we transform the parameters (10) as follows

$$
\begin{gathered}
\xi_{0}^{\prime}=\xi_{0}, \quad \boldsymbol{\xi}^{\prime}=R(U) \boldsymbol{\xi}, \\
\eta_{00}^{\prime}=\eta_{00}, \quad \boldsymbol{\eta}^{\prime}=R(U) \boldsymbol{\eta}, \\
E^{\prime}=R(U) E R^{\mathrm{T}}(U) .
\end{gathered}
$$

This can for instance be used to diagonalise the real symmetric matrix $E$.

The precise conditions for the potential (9) to be stable and to lead to the EWSB observed in Nature were spelled out in [16]. A thorough investigation of the possibilities for $\mathrm{CP}$ invariance and spontaneous $\mathrm{CP}$ violation for the Higgs Lagrangian (3) with the potential (9) was done in [17]. There, we gave a classification of possible generalised CP transformations of the Lagrangian (3) in type (i) and (ii). This classification is geometrically motivated: The $\mathrm{CP}_{g}^{(i)}$ transformation of type (i) corresponds to the point reflection at the origin in $\mathbf{K}$-space, whereas a type (ii) $\mathrm{CP}_{g}$ transformation corresponds to a reflection on a plane in $\mathbf{K}$-space. Note that it is not possible to convert type (i) and type (ii) transformations into each other by a change of basis.

In terms of the fields, the type (i) generalised CP transformation is denoted by $\mathrm{CP}_{g}^{(i)}$ and defined by (see (I.54))

$$
\begin{aligned}
\mathrm{CP}_{g}^{(i)}: W^{\mu}(x) & \rightarrow-W_{\mu}^{\mathrm{T}}\left(x^{\prime}\right), \\
B^{\mu}(x) & \rightarrow-B_{\mu}\left(x^{\prime}\right), \\
\varphi_{i}(x) & \rightarrow \epsilon_{i j} \varphi_{j}^{*}\left(x^{\prime}\right), \\
\phi(x) & \rightarrow \epsilon \phi^{*}\left(x^{\prime}\right) .
\end{aligned}
$$


Here

$$
x=\left(\begin{array}{c}
x^{0} \\
\mathbf{x}
\end{array}\right), \quad x^{\prime}=\left(\begin{array}{c}
x^{0} \\
-\mathbf{x}
\end{array}\right),
$$

and

$$
W^{\mu}(x)=W^{\mu a}(x) \frac{\tau^{a}}{2}
$$

is the matrix of $W$ four-potentials with $\tau^{a}(a=1,2,3)$ the Pauli matrices. Furthermore, $B^{\mu}(x)$ is the hypercharge four-potential and

$$
\epsilon=\left(\begin{array}{rr}
0 & 1 \\
-1 & 0
\end{array}\right)
$$

With (16) we find for the gauge-invariant functions

$$
\begin{aligned}
\mathrm{CP}_{g}^{(i)}: K_{0}(x) & \rightarrow K_{0}\left(x^{\prime}\right), \\
\mathbf{K}(x) & \rightarrow-\mathbf{K}\left(x^{\prime}\right) .
\end{aligned}
$$

That is, the $\mathrm{CP}_{g}^{(i)}$ transformation (16) corresponds to the point reflection at the origin in $\mathbf{K}$-space in addition to the change of argument $x \rightarrow x^{\prime}$. Twofold application of the $\mathrm{CP}_{g}^{(i)}$ transformation gives the original Higgs fields with a minus sign. This minus sign corresponds to a hypercharge transformation, and thus drops out when considering the twofold $\mathrm{CP}_{g}^{(i)}$ transformation (20) of the gauge-invariant functions. Note that the $\mathrm{CP}_{g}^{(i)}$ transformation of the fields given by (16) has the same form in any Higgs basis, up to gauge transformations. See section I.3 and appendix I.B.

The type (ii) generalised CP transformations are those which reduce to the standard $\mathrm{CP}$ transformation in a suitable basis for the Higgs doublets (see section I.3.2). That is, after a suitable basis change (11) we have

$$
\mathrm{CP}_{g}^{(i i)}: \varphi_{i}^{\prime}(x) \rightarrow \varphi_{i}^{\prime *}\left(x^{\prime}\right), \quad i \in\{1,2\},
$$

whereas the transformation of the gauge potentials stays as in (15). For the original Higgs basis we have then:

$$
\mathrm{CP}_{g}^{(i i)}: \varphi_{i}(x) \rightarrow\left(U^{-1} U^{*}\right)_{i j} \varphi_{j}{ }^{*}\left(x^{\prime}\right) .
$$

Here, the argument change and complex conjugation of the Higgs fields $\varphi_{i}(x)$ is supplemented by a basis change. For the gauge-invariant functions these type (ii) $\mathrm{CP}_{g}$ transformations (22) correspond to reflections on a plane in $\mathbf{K}$-space. These reflections are orthogonally equivalent to $R_{2}$, the reflection on the 1-3 plane. Indeed, let us define the reflections on the coordinate planes as

$$
\begin{aligned}
& R_{1}=\operatorname{diag}(-1,1,1), \\
& R_{2}=\operatorname{diag}(1,-1,1), \\
& R_{3}=\operatorname{diag}(1,1,-1) .
\end{aligned}
$$

Then we find from (22)

$$
\begin{aligned}
\mathrm{CP}_{g}^{(i i)}: \quad K_{0}(x) & \rightarrow K_{0}(x), \\
\mathbf{K}(x) & \rightarrow \bar{R}_{\varphi} \mathbf{K}\left(x^{\prime}\right),
\end{aligned}
$$

where we have with $R(U)$ from (13)

$$
\bar{R}_{\varphi}=R^{\mathrm{T}}(U) R_{2} R(U) .
$$

Note that a twofold $\mathrm{CP}_{g}^{(i i)}$ transformation reproduces the original Higgs fields without an additional phase.

Invariance of the potential (9) under a generalised CP transformation was found in section I.3 to require for the parameters (10)

$$
\bar{R} \boldsymbol{\xi}=\boldsymbol{\xi}, \quad \bar{R} \boldsymbol{\eta}=\boldsymbol{\eta}, \quad \bar{R} E \bar{R}^{\mathrm{T}}=E .
$$

Here $\bar{R}$ is the improper rotation matrix in $\mathbf{K}$-space corresponding to the generalised $\mathrm{CP}$ symmetry. A generalised $\mathrm{CP}_{g}$ symmetry of type (i) corresponds to $\bar{R}=-\mathbb{1}_{3}$ whereas type (ii) corresponds to $\bar{R}=\bar{R}_{\varphi}$, (25).

Now we can write down the most general potential having $\mathrm{CP}_{g}^{(i)}$ invariance; see theorem I.2. We must have $\boldsymbol{\xi}=0$ and $\boldsymbol{\eta}=0$ in order to satisfy (26). Thus,

$$
V=\xi_{0} K_{0}(x)+\eta_{00} K_{0}^{2}(x)+\mathbf{K}^{\mathrm{T}}(x) E \mathbf{K}(x) .
$$

In the following we shall always suppose that by a basis change as in (11) and (14) we have diagonalised $E$ and ordered the eigenvalues as follows:

$$
E=\operatorname{diag}\left(\mu_{1}, \mu_{2}, \mu_{3}\right), \quad \mu_{1} \geq \mu_{2} \geq \mu_{3} .
$$

In theorem I.5 we found that the potential (27) leads to a stable theory with the correct EWSB and no zero mass charged Higgs bosons if and only if

$$
\begin{aligned}
\eta_{00} & >0, \\
\mu_{a}+\eta_{00} & >0 \quad \text { for } a=1,2,3, \\
\xi_{0} & <0 \\
\mu_{3} & <0 .
\end{aligned}
$$

In the following we shall always suppose these conditions to hold.

\section{THE VACUUM EXPECTATION VALUES AND THE HIGGS MASS SPECTRUM}

The vacuum solution for the Higgs fields, denoted by

$$
\left\langle\varphi_{j}\right\rangle:=\left\langle\varphi_{j}(x)\right\rangle=\left(\begin{array}{c}
v_{j}^{+} \\
v_{j}^{0}
\end{array}\right), \quad j=1,2
$$

is obtained from the global minimum of $V$ (27). The corresponding matrices $\langle\phi\rangle$ and $\underline{K}$ are

$$
\begin{gathered}
\langle\phi\rangle:=\langle\phi(x)\rangle=\left(\begin{array}{ll}
v_{1}^{+} & v_{1}^{0} \\
v_{2}^{+} & v_{2}^{0}
\end{array}\right), \\
\underline{K}=\langle\phi\rangle\langle\phi\rangle^{\dagger}=\frac{1}{2}\left(K_{0} \mathbb{1}_{2}+\mathbf{K} \boldsymbol{\sigma}\right), \\
\tilde{\mathbf{K}}=\left(\begin{array}{c}
K_{0} \\
\mathbf{K}
\end{array}\right) .
\end{gathered}
$$


In appendix I.B we have already discussed this vacuum solution. We get (see (I.B.41))

$$
\tilde{\mathbf{K}}=\left(\begin{array}{c}
K^{0} \\
\mathbf{K}
\end{array}\right)=\frac{-\xi_{0}}{2\left(\eta_{00}+\mu_{3}\right)}\left(\begin{array}{l}
1 \\
0 \\
0 \\
1
\end{array}\right)
$$

In section 7 of [16] a general discussion of the structure of the Higgs sector in THDMs after EWSB was given. The basis choice in this reference coincides with our present choice. We can, therefore, set for the Higgs field vacuum expectation values

$$
\begin{aligned}
\left\langle\varphi_{1}\right\rangle & =\frac{1}{\sqrt{2}}\left(\begin{array}{c}
0 \\
v_{0}
\end{array}\right), \\
\left\langle\varphi_{2}\right\rangle & =\left(\begin{array}{l}
0 \\
0
\end{array}\right), \\
\langle\phi\rangle & =\frac{1}{\sqrt{2}}\left(\begin{array}{cc}
0 & v_{0} \\
0 & 0
\end{array}\right) .
\end{aligned}
$$

Here

$$
v_{0} \approx 246 \mathrm{GeV}
$$

is the standard Higgs vacuum expectation value (see for instance [4]). Furthermore, we use the unitary gauge and introduce the shifted, that is, the physical Higgs fields as in (129) $\mathrm{ff}$ of [16]. This leads to

$$
\begin{aligned}
\varphi_{1}(x) & =\frac{1}{\sqrt{2}}\left(\begin{array}{c}
0 \\
v_{0}+\rho^{\prime}(x)
\end{array}\right), \\
\varphi_{2}(x) & =\left(\begin{array}{c}
H^{+}(x) \\
\frac{1}{\sqrt{2}}\left(h^{\prime}(x)+i h^{\prime \prime}(x)\right)
\end{array}\right) .
\end{aligned}
$$

Here $\rho^{\prime}(x), h^{\prime}(x)$ and $h^{\prime \prime}(x)$ are the three real fields corresponding to the physical neutral Higgs particles and $\mathrm{H}^{+}(x)$ is the complex field corresponding to the physical charged Higgs particle. We set

$$
H^{-}(x)=\left(H^{+}(x)\right)^{*} \text {. }
$$

From the results of section 7 of [16] and appendix I.B we can now immediately read off a number of relations.

The Lagrange multiplier $u_{0}$ corresponding to the global minimum of the potential $V$ (27) is

$$
u_{0}=-\mu_{3} \text {. }
$$

Inserting (35) in (32) we get for the vacuum four-vector $\tilde{\mathbf{K}}(\operatorname{see}(134)$ of [16])

$$
\tilde{\mathbf{K}}=\frac{1}{2} v_{0}^{2}\left(\begin{array}{l}
1 \\
0 \\
0 \\
1
\end{array}\right) .
$$

Comparison with (34) gives

$$
\begin{aligned}
& v_{0}^{2}=\frac{-\xi_{0}}{\eta_{00}+\mu_{3}}=\frac{\left|\xi_{0}\right|}{\eta_{00}-\left|\mu_{3}\right|}, \\
& v_{0}=\sqrt{\frac{-\xi_{0}}{\eta_{00}+\mu_{3}}} .
\end{aligned}
$$

The mass squared of the charged Higgs particles is, according to (145) of [16],

$$
\begin{aligned}
m_{H^{ \pm}}^{2} & =2 u_{0} v_{0}^{2}=2\left(-\mu_{3}\right) v_{0}^{2} \\
& =\frac{2 \mu_{3} \xi_{0}}{\eta_{00}+\mu_{3}} .
\end{aligned}
$$

The mass matrix squared of the neutral Higgs particles is obtained from (144) of [16] as follows:

$$
\mathcal{M}_{\text {neutral }}^{2}=2\left(\begin{array}{ccc}
-\xi_{0} & 0 & 0 \\
0 & v_{0}^{2}\left(\mu_{1}-\mu_{3}\right) & 0 \\
0 & 0 & v_{0}^{2}\left(\mu_{2}-\mu_{3}\right)
\end{array}\right)
$$

with the ordering $\left(\rho^{\prime}, h^{\prime}, h^{\prime \prime}\right)$ for the fields. We see that $\mathcal{M}_{\text {neutral }}^{2}$ is already diagonal with our choice of basis. Thus we have

$$
\begin{aligned}
m_{\rho^{\prime}}^{2} & =2\left(-\xi_{0}\right), \\
m_{h^{\prime}}^{2} & =2 v_{0}^{2}\left(\mu_{1}-\mu_{3}\right), \\
m_{h^{\prime \prime}}^{2} & =2 v_{0}^{2}\left(\mu_{2}-\mu_{3}\right) .
\end{aligned}
$$

In the following we shall require that none of the neutral physical Higgs particles is massless and that there is no mass degeneracy between $h^{\prime}$ and $h^{\prime \prime}$. This implies from (45) the condition

$$
\mu_{1}>\mu_{2}>\mu_{3}
$$

which is slightly stricter than (28).

Our Higgs potential (27) has five parameters $\xi_{0}, \eta_{00}$, $\mu_{1}, \mu_{2}, \mu_{3}$. We can now express these in terms of the five physical quantities $v_{0}^{2}, m_{H^{ \pm}}^{2}, m_{\rho^{\prime}}^{2}, m_{h^{\prime}}^{2}, m_{h^{\prime \prime}}^{2}$. This gives

$$
\begin{aligned}
\xi_{0} & =-\frac{1}{2} m_{\rho^{\prime}}^{2} \\
\eta_{00} & =\frac{1}{2 v_{0}^{2}}\left(m_{H^{ \pm}}^{2}+m_{\rho^{\prime}}^{2}\right), \\
\mu_{1} & =\frac{1}{2 v_{0}^{2}}\left(m_{h^{\prime}}^{2}-m_{H^{ \pm}}^{2}\right), \\
\mu_{2} & =\frac{1}{2 v_{0}^{2}}\left(m_{h^{\prime \prime}}^{2}-m_{H^{ \pm}}^{2}\right), \\
\mu_{3} & =-\frac{1}{2 v_{0}^{2}} m_{H^{ \pm}}^{2} .
\end{aligned}
$$

For positive squared masses and $m_{h^{\prime}}^{2}>m_{h^{\prime \prime}}^{2}$ the conditions (29) and (46) are always satisfied.

Let us next discuss the CP symmetries of our model and the CP transformation properties of the vacuum expectation values and of the physical fields. The Higgs Lagrangian (3) with the potential (27) for which we require (46) to hold, allows for exactly four generalised CP transformations, $\mathrm{CP}_{g}^{(i)}, \mathrm{CP}_{g, 1}^{(i i)}, \mathrm{CP}_{g, 2}^{(i i)}$ and $\mathrm{CP}_{g, 3}^{(i i)}$ as defined below. In all cases the gauge potentials are transformed according to (15). But the transformation of the Higgs fields and of the gauge-invariant functions $K_{0}(x)$, $\mathbf{K}(x)$ is different. 
Our basic type $(i) \mathrm{CP}$ transformation, $\mathrm{CP}_{g}^{(i)}$, transforms the Higgs fields and the gauge-invariant functions according to (16) and (20), respectively.

For the type (ii) transformation $\mathrm{CP}_{g, 1}^{(i i)}$ we set

$$
\begin{aligned}
\mathrm{CP}_{g, 1}^{(i i)}: & \varphi_{i}(x) \rightarrow \sigma_{i j}^{3} \varphi_{j}^{*}\left(x^{\prime}\right) \\
& \varphi_{1}(x) \rightarrow \varphi_{1}^{*}\left(x^{\prime}\right) \\
& \varphi_{2}(x) \rightarrow-\varphi_{2}^{*}\left(x^{\prime}\right)
\end{aligned}
$$

This implies

$$
\begin{aligned}
\mathrm{CP}_{g, 1}^{(i i)}: \quad K_{0}(x) & \rightarrow K_{0}\left(x^{\prime}\right), \\
\mathbf{K}(x) & \rightarrow R_{1} \mathbf{K}\left(x^{\prime}\right),
\end{aligned}
$$

with $R_{1}$ the matrix of the reflection on the $2-3$ plane; see (23).

The type (ii) transformation $\mathrm{CP}_{g, 2}^{(i i)}$ is the standard $\mathrm{CP}$ transformation, $\mathrm{CP}_{s}$, for the Higgs fields, where

$$
\begin{aligned}
\mathrm{CP}_{g, 2}^{(i i)}: & \varphi_{1}(x) \rightarrow \varphi_{1}^{*}\left(x^{\prime}\right), \\
& \varphi_{2}(x) \rightarrow \varphi_{2}^{*}\left(x^{\prime}\right) .
\end{aligned}
$$

Here we get

$$
\begin{aligned}
\mathrm{CP}_{g, 2}^{(i i)}: \quad K_{0}(x) & \rightarrow K_{0}\left(x^{\prime}\right), \\
\mathbf{K}(x) & \rightarrow R_{2} \mathbf{K}\left(x^{\prime}\right),
\end{aligned}
$$

with $R_{2}$ the matrix of the reflection on the $1-3$ plane; see (23).

Finally, the transformation $\mathrm{CP}_{g, 3}^{(i i)}$ is defined by

$$
\begin{aligned}
\mathrm{CP}_{g, 3}^{(i i)}: & \varphi_{i}(x) \rightarrow \sigma_{i j}^{1} \varphi_{j}^{*}\left(x^{\prime}\right) \\
& \varphi_{1}(x) \rightarrow \varphi_{2}^{*}\left(x^{\prime}\right) \\
& \varphi_{2}(x) \rightarrow \varphi_{1}^{*}\left(x^{\prime}\right)
\end{aligned}
$$

This implies

$$
\begin{aligned}
\mathrm{CP}_{g, 3}^{(i i)}: \quad K_{0}(x) & \rightarrow K_{0}\left(x^{\prime}\right), \\
\mathbf{K}(x) & \rightarrow R_{3} \mathbf{K}\left(x^{\prime}\right),
\end{aligned}
$$

with $R_{3}$ the reflection on the 1-2 plane; see (23).

Now we summarise the four different $\mathrm{CP}_{g}$ transformations for the Higgs fields as

$$
\mathrm{CP}_{g}: \quad \varphi_{i}(x) \rightarrow W_{i j} \varphi_{j}^{*}\left(x^{\prime}\right) .
$$

The matrices $W=\left(W_{i j}\right)$ for the various $\mathrm{CP}_{g}$ transformations are listed in Tab. (1) see (16), (48), (501) and (52). We note that we could supplement an additional global phase factor on the right-hand side of (54). However, such a global phase factor in the Higgs CP transformation drops out in the Higgs potential, and for the Yukawa terms it may always be absorbed by proper redefinitions of the fermion fields, as will be explained in the next section. Therefore we may without loss of generality set this global phase factor to 1 .

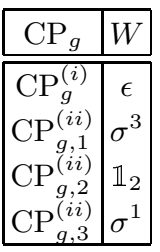

TABLE I: The matrices $W$ (54) for the four $\mathrm{CP}_{g}$ transformations.

Note that the symmetries $\mathrm{CP}_{g}^{(i)}, \mathrm{CP}_{g, 1}^{(i i)}, \mathrm{CP}_{g, 2}^{(i i)}$ and $\mathrm{CP}_{g, 3}^{(i i)}$ are not independent since we have at the level of the transformation of the Higgs fields the relation

$$
\mathrm{CP}_{g, 3}^{(i i)}=\mathrm{CP}_{g, 1}^{(i i)} \circ \mathrm{CP}_{g, 2}^{(i i)} \circ \mathrm{CP}_{g}^{(i)} .
$$

Any of the above CP symmetries is conserved by the vacuum if and only if the vacuum value $\mathbf{K}$ satisfies

$$
\bar{R} \mathbf{K}=\mathbf{K} \text {. }
$$

Here we have to insert $\bar{R}=-\mathbb{1}_{3}, R_{1}, R_{2}$ and $R_{3}$ for the symmetries $\mathrm{CP}_{g}^{(i)}, \mathrm{CP}_{g, 1}^{(i i)} \mathrm{CP}_{g, 2}^{(i i)}$ and $\mathrm{CP}_{g, 3}^{(i i)}$, respectively. Looking at the vacuum solution (34) for $\tilde{\mathbf{K}}$ we see immediately that

$$
\begin{aligned}
\left(-\mathbb{1}_{3}\right) \mathbf{K} & \neq \mathbf{K}, \\
R_{1} \mathbf{K} & =\mathbf{K}, \\
R_{2} \mathbf{K} & =\mathbf{K}, \\
R_{3} \mathbf{K} & \neq \mathbf{K} .
\end{aligned}
$$

Thus, the symmetries $\mathrm{CP}_{g}^{(i)}$ and $\mathrm{CP}_{g, 3}^{(i i)}$ are spontaneously broken, as we already know from theorem I.4. On the other hand, the symmetries $\mathrm{CP}_{g, 1}^{(i i)}$ and $\mathrm{CP}_{g, 2}^{(i i)}$ are conserved by the vacuum.

Now we come to the CP transformation properties of the physical Higgs fields defined in (37) and (38). Under $\mathrm{CP}_{g}^{(i)}$, which transforms the Higgs doublets according to (16), the physical Higgs fields have no definite transformation property. This is alright, since $\mathrm{CP}_{g}^{(i)}$ is spontaneously broken. For the unbroken symmetry $\mathrm{CP}_{g, 1}^{(i i)}$ we get from (37), (38) and (48)

$$
\begin{aligned}
\mathrm{CP}_{g, 1}^{(i i)}: \quad \rho^{\prime}(x) & \rightarrow \rho^{\prime}\left(x^{\prime}\right), \\
h^{\prime}(x) & \rightarrow-h^{\prime}\left(x^{\prime}\right), \\
h^{\prime \prime}(x) & \rightarrow h^{\prime \prime}\left(x^{\prime}\right), \\
H^{+}(x) & \rightarrow-H^{-}\left(x^{\prime}\right) .
\end{aligned}
$$

On the other hand, we obtain from (37), (38) and (50) for the $\mathrm{CP}_{g, 2}^{(i i)}$ symmetry

$$
\begin{aligned}
\mathrm{CP}_{g, 2}^{(i i)}: \quad \rho^{\prime}(x) & \rightarrow \rho^{\prime}\left(x^{\prime}\right), \\
h^{\prime}(x) & \rightarrow h^{\prime}\left(x^{\prime}\right), \\
h^{\prime \prime}(x) & \rightarrow-h^{\prime \prime}\left(x^{\prime}\right), \\
H^{+}(x) & \rightarrow H^{-}\left(x^{\prime}\right) .
\end{aligned}
$$




\begin{tabular}{|c|c|c|}
\hline Fermion & weak isospin $t$ & weak hypercharge $y$ \\
\hline \hline$\left(\begin{array}{c}\nu_{j L} \\
l_{j L}\end{array}\right)$ & $1 / 2$ & $-1 / 2$ \\
\hline$\nu_{j R}$ & 0 & 0 \\
\hline$l_{j R}$ & 0 & -1 \\
\hline$\left(\begin{array}{c}u_{j L} \\
d_{j L}\end{array}\right)$ & $1 / 2$ & $1 / 6$ \\
\hline$u_{j R}$ & 0 & $2 / 3$ \\
\hline$d_{j R}$ & 0 & $-1 / 3$ \\
\hline
\end{tabular}

TABLE II: The fermion families, $j=1,2,3$, and their quantum numbers of weak isospin $t$ and weak hypercharge $y$.

We see that the field $\rho^{\prime}$ is $\mathrm{CP}_{g, 1}^{(i i)}$ and $\mathrm{CP}_{g, 2}^{(i i)}$ even, $h^{\prime}$ is $\mathrm{CP}_{g, 1}^{(i i)}$ odd and $h^{\prime \prime}$ is $\mathrm{CP}_{g, 1}^{(i i)}$ even. This role of $h^{\prime}$ and $h^{\prime \prime}$ is interchanged for the symmetry $\mathrm{CP}_{g, 2}^{(i i)}$; see (59). We note, however, that this assignment of $\mathrm{CP}_{g}^{(i i)}$ quantum numbers is to some extent a convention, since we could have inserted extra global factors of $(-1)$ in (48) and (50). This would not change the transformation properties of the gauge-invariant functions in (49) and (51) and thus have no physical consequence.

\section{FERMIONS AND THEIR COUPLINGS TO THE HIGGS FIELDS}

In this section we shall discuss the fermion families and their coupling to the Higgs fields. We shall require that the Higgs-fermion coupling, that is the Yukawa term $\mathscr{L}_{\text {Yuk }}$ in (2), respects all four generalised CP symmetries, $\mathrm{CP}_{g}^{(i)}, \mathrm{CP}_{g, 1}^{(i i)}, \mathrm{CP}_{g, 2}^{(i i)}$ and $\mathrm{CP}_{g, 3}^{(i i)}$, as introduced in section 3 We shall call this the "principle of maximal CP invariance". We shall show that this principle leads to interesting consequences.

Let us first introduce our notation for the fermions; see Tab. II We give the fermions a family index $j(j=$ $1,2,3)$ for ease of notation. Thus, we set $l_{1} \equiv e, l_{2} \equiv \mu$ and $l_{3} \equiv \tau$ for the leptons, $u_{1} \equiv u, u_{2} \equiv c, u_{3} \equiv t$ for the up type quarks and $d_{1} \equiv d, d_{2} \equiv s$ and $d_{3} \equiv b$ for the down type quarks. The indices $L$ and $R$ stand for left- and right-handed fields, respectively. In Tab. [II we list also right-handed neutrinos. The finding of neutrino oscillations suggests that they also form part of Nature. In the following, however, we shall, as an approximation, consider the neutrinos as massless and ignore the $\nu_{j} R$ fields.

\subsection{The case of one family}

Now we study if we can couple one fermion family to the Higgs doublets in a $\mathrm{CP}_{g}^{(i)}$-invariant way. The most general $S U(2)_{L} \times U(1)_{Y}$ invariant Yukawa interaction for this case has the form (see chapter 22.4 of [4] for the analogous discussion in the framework of the SM)

$$
\begin{aligned}
\mathscr{L}_{\text {Yuk }}(x)= & -\bar{l}_{1 R}(x) c_{l, i} \varphi_{i}^{\dagger}(x)\left(\begin{array}{c}
\nu_{1 L}(x) \\
l_{1 L}(x)
\end{array}\right) \\
& +\bar{u}_{1 R}(x) c_{q, i}^{\prime} \varphi_{i}^{\mathrm{T}}(x) \epsilon\left(\begin{array}{c}
u_{1 L}(x) \\
d_{1 L}(x)
\end{array}\right) \\
& -\bar{d}_{1 R}(x) c_{q, i} \varphi_{i}^{\dagger}(x)\left(\begin{array}{c}
u_{1 L}(x) \\
d_{1 L}(x)
\end{array}\right) \\
& + \text { h.c. }
\end{aligned}
$$

Here $c_{l, i}, c_{q, i}^{\prime}$ and $c_{q, i}(i=1,2)$ are arbitrary complex numbers.

Now we make a general ansatz for the $\mathrm{CP}_{g}^{(i)}$ transformation of the fermion fields as follows:

$$
\begin{aligned}
\left(\begin{array}{c}
\nu_{1 L}(x) \\
l_{1 L}(x)
\end{array}\right) & \rightarrow e^{i \xi_{1}} \gamma^{0} S(C)\left(\begin{array}{l}
\bar{\nu}_{1 L}^{\mathrm{T}}\left(x^{\prime}\right) \\
\bar{l}_{1 L}^{\mathrm{T}}\left(x^{\prime}\right)
\end{array}\right), \\
l_{1 R}(x) & \rightarrow e^{i \xi_{2}} \gamma^{0} S(C) \bar{l}_{1 R}^{\mathrm{T}}\left(x^{\prime}\right), \\
\left(\begin{array}{c}
u_{1 L}(x) \\
d_{1 L}(x)
\end{array}\right) & \rightarrow e^{i \xi_{3}} \gamma^{0} S(C)\left(\begin{array}{l}
\bar{u}_{1 L}^{\mathrm{T}}\left(x^{\prime}\right) \\
\bar{d}_{1 L}^{\mathrm{T}}\left(x^{\prime}\right)
\end{array}\right), \\
u_{1 R}(x) & \rightarrow e^{i \xi_{4}} \gamma^{0} S(C) \bar{u}_{1 R}^{\mathrm{T}}\left(x^{\prime}\right), \\
d_{1 R}(x) & \rightarrow e^{i \xi_{5}} \gamma^{0} S(C) \bar{d}_{1 R}^{\mathrm{T}}\left(x^{\prime}\right) .
\end{aligned}
$$

Here $x, x^{\prime}$ are as in (17) and $\gamma^{0}$ and $S(C):=i \gamma^{2} \gamma^{0}$ are the usual Dirac matrices for the parity and charge conjugation transformations, respectively (see for instance chapter 4 of [4]). For generality we have inserted in (61) arbitrary phase factors $e^{i \xi_{a}}$ with $\xi_{a}(a=1, \ldots, 5)$ real. With (16) and (61) we find the following transformation of $\mathscr{L}_{\text {Yuk }}(x)$, (60):

$$
\begin{aligned}
& \mathscr{L}_{\mathrm{Yuk}}(x) \rightarrow \\
&- e^{i\left(\xi_{1}-\xi_{2}\right)} \times \\
&\left(\bar{\nu}_{1 L}\left(x^{\prime}\right), \bar{l}_{1 L}\left(x^{\prime}\right)\right) c_{l, j} \epsilon_{j i} \varphi_{i}\left(x^{\prime}\right) l_{1 R}\left(x^{\prime}\right) \\
&+ e^{i\left(\xi_{3}-\xi_{4}\right)} \times \\
&\left(\bar{u}_{1 L}\left(x^{\prime}\right), \bar{d}_{1 L}\left(x^{\prime}\right)\right) c_{q, j}^{\prime} \epsilon_{j i} \epsilon^{\mathrm{T}} \varphi_{i}^{*}\left(x^{\prime}\right) u_{1 R}\left(x^{\prime}\right) \\
&- e^{i\left(\xi_{3}-\xi_{5}\right)} \times \\
&\left(\bar{u}_{1 L}\left(x^{\prime}\right), \bar{d}_{1 L}\left(x^{\prime}\right)\right) c_{q, j} \epsilon_{j i} \varphi_{i}\left(x^{\prime}\right) d_{1 R}\left(x^{\prime}\right) \\
&+ \text { h.c. }
\end{aligned}
$$

Note that a possible additional global phase factor in the CP transformation of the Higgs fields, that is, on the right-hand side of (16), can be absorbed by a redefinition of the phases $\xi_{2}, \xi_{4}$ and $\xi_{5}$ for the right-handed fermions. Similar remarks apply to the case of more than one fermion family. Comparing (62) with (60) we see that we have $\mathrm{CP}_{g}^{(i)}$ invariance, $\mathscr{L}_{\text {Yuk }}(x) \rightarrow \mathscr{L}_{\text {Yuk }}\left(x^{\prime}\right)$, if 
and only if

$$
\begin{aligned}
c_{l, i}^{*} & =e^{i\left(\xi_{1}-\xi_{2}\right)} c_{l, j} \epsilon_{j i}, \\
c_{q, i}^{\prime} & =e^{i\left(\xi_{3}-\xi_{4}\right)} c_{q, j}^{\prime} \epsilon_{j i}, \\
c_{q, i}^{*} & =e^{i\left(\xi_{3}-\xi_{5}\right)} c_{q, j} \epsilon_{j i}
\end{aligned}
$$

for $i=1,2$. Explicitly we find from (63) for $c_{l, i}$ :

$$
\begin{aligned}
& c_{l, 1}^{*}=-e^{i\left(\xi_{1}-\xi_{2}\right)} c_{l, 2}, \\
& c_{l, 2}^{*}=e^{i\left(\xi_{1}-\xi_{2}\right)} c_{l, 1},
\end{aligned}
$$

which has only the trivial solution

$$
c_{l, 1}=c_{l, 2}=0 .
$$

The same result is found for $c_{q, i}$ and $c_{q, i}^{\prime}$.

We summarise these findings as follows. A single fermion family (see Tab. II) cannot be coupled to the two-Higgs-doublet fields in a $\mathrm{CP}_{g}^{(i)}$-symmetric way. In other words: requiring $\mathrm{CP}_{g}^{(i)}$ symmetry for the Yukawa Lagrangian (60) leads to

$$
c_{l, i}=c_{q, i}=c_{q, i}^{\prime}=0, \quad i=1,2,
$$

that is, to $\mathscr{L}_{\text {Yuk }} \equiv 0$.

Our principle of maximal $\mathrm{CP}$ invariance has as part of its requirements $\mathrm{CP}_{g}^{(i)}$ symmetry. Thus, we have shown that a single fermion family cannot be coupled to the Higgs doublets in a way respecting our principle.

\subsection{The case of two families, generalities}

In this section we shall treat the case of two families where, for definiteness, we consider the families 2 and 3. The most general Yukawa interaction of these families with the Higgs doublets can be written as

$$
\begin{aligned}
\mathscr{L}_{\text {Yuk }}(x)= & -\bar{l}_{\alpha R}(x) C_{l \alpha \beta}^{(j)} \varphi_{j}^{\dagger}(x)\left(\begin{array}{c}
\nu_{\beta L}(x) \\
l_{\beta L}(x)
\end{array}\right) \\
& +\bar{u}_{\alpha R}(x) C_{q \alpha \beta}^{(j)} \varphi_{j}^{\mathrm{T}}(x) \epsilon\left(\begin{array}{c}
u_{\beta L}(x) \\
d_{\beta L}^{\prime}(x)
\end{array}\right) \\
& -\bar{d}_{\alpha R}^{\prime}(x) C_{q \alpha \beta}^{(j)} \varphi_{j}^{\dagger}(x)\left(\begin{array}{c}
u_{\beta L}(x) \\
d_{\beta L}^{\prime}(x)
\end{array}\right) \\
& + \text { h.c. }
\end{aligned}
$$

Here $\alpha, \beta \in\{2,3\}$ are the family indices and $j \in\{1,2\}$ number the Higgs doublets. The summation convention is used if not stated otherwise. The $2 \times 2$ matrices $C_{l}^{(j)}=$ $\left(C_{l \alpha \beta}^{(j)}\right), C_{q}^{(j)}=\left(C_{q \alpha \beta}^{(j)}\right), C_{q}^{\prime(j)}=\left(C_{q \alpha \beta}^{\prime(j)}\right)$ have, to start with, arbitrary complex entries.

Without changing the physical content of the theory we can make $U(2)$-rotations of the right-handed fields $l_{\alpha R}$, $u_{\alpha R}, d_{\alpha R}^{\prime}$ and the left-handed doublet fields $\left(\nu_{\alpha L}, l_{\alpha L}\right)^{\mathrm{T}}$ and $\left(u_{\alpha L}, d_{\alpha L}^{\prime}\right)^{\mathrm{T}}$. As in the SM we can use this to require, without loss of generality, for the matrices $C_{l}^{(1)}$, $C_{q}^{(1)}$ and $C_{q}^{(1)}$ certain standard forms:

$$
\begin{aligned}
C_{l}^{(1)} & =\left(\begin{array}{cc}
c_{l 2}^{(1)} & 0 \\
0 & c_{l 3}^{(1)}
\end{array}\right), & & c_{l 2}^{(1)} \geq 0, \quad c_{l 3}^{(1)} \geq 0 ; \\
C_{q}^{(1)} & =\left(\begin{array}{cc}
c_{u 2}^{(1)} & 0 \\
0 & c_{u 3}^{(1)}
\end{array}\right), & & c_{u 2}^{(1)} \geq 0, \quad c_{u 3}^{(1)} \geq 0 ; \\
C_{q}^{(1)} & =V\left(\begin{array}{cc}
c_{d 2}^{(1)} & 0 \\
0 & c_{d 3}^{(1)}
\end{array}\right) V^{\dagger}, & & c_{d 2}^{(1)} \geq 0, \quad c_{d 3}^{(1)} \geq 0 \\
V & =\left(\begin{array}{cc}
\cos \vartheta & \sin \vartheta \\
-\sin \vartheta & \cos \vartheta
\end{array}\right), & & 0 \leq \vartheta \leq \pi / 2 .
\end{aligned}
$$

For the derivation of the corresponding results in the SM see, for instance, chapter 22.4 of [4]. The matrix $V=$ $\left(V_{\alpha \beta}\right)$ in (71) will turn out to be the Cabibbo-KobayashiMaskawa (CKM) matrix in the 2-3 sector. As we shall see, in the basis of the fermion fields defined by (68)-(71) the fields $l_{\alpha R}, l_{\alpha L}$ and $u_{\alpha R}, u_{\alpha L}$ correspond to mass eigenfields. For the $d^{\prime}$-fields defined in this basis the mass eigenstates will be

$$
d_{\alpha R, L}(x)=V_{\alpha \beta}^{\dagger} d_{\beta R, L}^{\prime}(x) .
$$

In the following we shall always work in the fermion basis defined by (68)-(71) if not stated otherwise.

For the $\mathrm{CP}_{g}$ transformations of the fermions we make the generic ansatz:

$$
\begin{aligned}
\mathrm{CP}_{g}:\left(\begin{array}{c}
\nu_{\alpha L}(x) \\
l_{\alpha L}(x)
\end{array}\right) & \rightarrow U_{L \alpha \beta}^{(l)} \gamma^{0} S(C)\left(\begin{array}{c}
\bar{\nu}_{\beta}^{\mathrm{T}}\left(x^{\prime}\right) \\
\bar{l}_{\beta L}^{\mathrm{T}}\left(x^{\prime}\right)
\end{array}\right), \\
l_{\alpha R}(x) & \rightarrow U_{R \alpha \beta}^{(l)} \gamma^{0} S(C) \bar{l}_{\beta R}^{\mathrm{T}}\left(x^{\prime}\right), \\
\left(\begin{array}{c}
u_{\alpha L}(x) \\
d_{\alpha L}^{\prime}(x)
\end{array}\right) & \rightarrow U_{L \alpha \beta}^{(u)} \gamma^{0} S(C)\left(\begin{array}{c}
\bar{u}_{\beta L}^{\mathrm{T}}\left(x^{\prime}\right) \\
\bar{d}_{\beta L}^{\mathrm{T}}\left(x^{\prime}\right)
\end{array}\right), \\
u_{\alpha R}(x) & \rightarrow U_{R \alpha \beta}^{(u)} \gamma^{0} S(C) \bar{u}_{\beta R}^{\mathrm{T}}\left(x^{\prime}\right), \\
d_{\alpha R}^{\prime}(x) & \rightarrow U_{R \alpha \beta}^{(d)} \gamma^{0} S(C) \bar{d}_{\beta R}^{\prime \mathrm{T}}\left(x^{\prime}\right) .
\end{aligned}
$$

All matrices $U_{L}^{(l)}=\left(U_{L \alpha \beta}^{(l)}\right), \ldots, U_{R}^{(d)}=\left(U_{R \alpha \beta}^{(d)}\right)$ are supposed to be unitary

$$
U_{L}^{(l)} U_{L}^{(l) \dagger}=\cdots=U_{R}^{(d)} U_{R}^{(d) \dagger}=\mathbb{1}_{2} .
$$

Of course, these matrices $U_{L}^{(l)}$ etc. will, in general, all be different for the four $\mathrm{CP}_{g}$ transformations which we consider.

Now we shall require that a $\mathrm{CP}_{g}$ transformation applied twice gives the original fields up to a phase factor. Writing generically for any of the transformations (73)

$$
\mathrm{CP}_{g}: \psi_{\alpha}(x) \rightarrow U_{\alpha \beta} \gamma^{0} S(C) \bar{\psi}_{\beta}^{\mathrm{T}}\left(x^{\prime}\right)
$$

we get

$$
\mathrm{CP}_{g} \circ \mathrm{CP}_{g}: \psi_{\alpha}(x) \rightarrow-\left(U U^{*}\right)_{\alpha \beta} \psi_{\beta}(x) .
$$


We shall, therefore, require

$$
-U U^{*}=e^{i \varkappa} \mathbb{1}_{2}
$$

with real $\varkappa$. In appendix $\mathrm{A}$ we show that there are only two types of solutions of (77).

$$
\begin{aligned}
\text { Type (a): } e^{i \varkappa} & =1, \\
U U^{*} & =-\mathbb{1}_{2}, \\
U & =e^{i \xi} \epsilon=e^{i \xi}\left(\begin{array}{cc}
0 & 1 \\
-1 & 0
\end{array}\right) .
\end{aligned}
$$

$$
\begin{aligned}
\text { Type (b) : } e^{i \varkappa} & =-1, \\
U U^{*} & =\mathbb{1}_{2}, \\
U & =e^{i \xi}\left(\begin{array}{cc}
\alpha & \beta \\
\beta & -\alpha^{*}
\end{array}\right), \\
\beta & \geq 0, \quad|\alpha|^{2}+\beta^{2}=1 .
\end{aligned}
$$

This classification of the fermion generation mixings in the CP transformations in type (a) and (b) is similar to the Higgs sector, where we distinguish the $\mathrm{CP}_{g}$ transformations of type (i) and (ii) according to the type of Higgs flavour mixing involved. If only the "standard" mixing types (ii) respectively (b) occur in a $\mathrm{CP}_{g}$ transformation, the operator $\left(\mathrm{CP}_{g}\right)^{2}$ is normalised as for the standard $\mathrm{CP}_{s}$ transformation. For certain combinations involving the "non-standard" mixing types (i) respectively (a), the operator $\left(\mathrm{CP}_{g}\right)^{2} \circ \exp (i 6 \pi Y)$ is normalised in the usual way, with $Y$ being the hypercharge operator; in other cases additional unobservable minus signs may occur for the fermions.

Let us next note the change of the matrices $U_{R, L}$ of (73) under a basis change of the fermion fields. Consider for instance a basis change of the $d_{R}$ fields as in (72):

$$
d_{\alpha R}(x)=V_{\alpha \beta}^{\dagger} d_{\beta R}^{\prime}(x)
$$

with $V=\left(V_{\alpha \beta}\right) \in U(2)$. The $\mathrm{CP}_{g}$ transformation of $d_{\alpha R}$ following from (73) is then

$$
d_{\alpha R}(x) \rightarrow \tilde{U}_{R \alpha \beta}^{(d)} \gamma^{0} S(C) \vec{d}_{\beta R}^{\mathrm{T}}\left(x^{\prime}\right)
$$

with

$$
\tilde{U}_{R}^{(d)}=V^{\dagger} U_{R}^{(d)} V^{*}
$$

It is easy to check that this transformation does not change the type of the $\mathrm{CP}_{g}$ transformation as described by (78) respectively (79).

For changes of basis of the other fermion fields the corresponding $U$ matrices transform analogously to (82).

Finally we consider a generic coupling of a fermion doublet field $\psi_{L \alpha}(x)$ and singlet field $\chi_{R \alpha}(x)$ to the Higgs fields:

$$
\mathscr{L}^{\prime}(x)=-\bar{\chi}_{\alpha R}(x) C_{\alpha \beta}^{(i)} \varphi_{i}^{\dagger}(x) \psi_{\beta L}(x)+\text { h.c. }
$$

A generic $\mathrm{CP}_{g}$ transformation for the Higgs fields as in (54) and for the fermions according to

$$
\begin{aligned}
\mathrm{CP}_{g}: & \psi_{\alpha L}(x) \rightarrow U_{L \alpha \beta}^{(\psi)} \gamma^{0} S(C) \bar{\psi}_{\beta L}^{\mathrm{T}}\left(x^{\prime}\right), \\
\chi_{\alpha R}(x) & \rightarrow U_{R \alpha \beta}^{(\chi)} \gamma^{0} S(C) \bar{\chi}_{\beta R}^{\mathrm{T}}\left(x^{\prime}\right)
\end{aligned}
$$

leads to

$$
\begin{aligned}
\mathrm{CP}_{g}: \mathscr{L}^{\prime}(x) \rightarrow & -\bar{\chi}_{\alpha R}\left(x^{\prime}\right) \tilde{C}_{\alpha \beta}^{(i)} \varphi_{i}^{\dagger}\left(x^{\prime}\right) \psi_{\beta L}\left(x^{\prime}\right) \\
& + \text { h.c. }
\end{aligned}
$$

where

$$
\tilde{C}^{(i)}=U_{R}^{(\chi) \mathrm{T}} C^{(j) *} U_{L}^{(\psi) *} W_{j i}
$$

Similarly we find for a coupling

$$
\mathscr{L}^{\prime \prime}(x)=\bar{\chi}_{\alpha R}(x) C_{\alpha \beta}^{\prime(i)} \varphi_{i}^{\mathrm{T}}(x) \epsilon \psi_{\beta L}(x)+h . c .
$$

the transformation

$$
\begin{aligned}
\mathrm{CP}_{g}: \mathscr{L}^{\prime \prime}(x) \rightarrow & \bar{\chi}_{\alpha R}\left(x^{\prime}\right) \tilde{C}_{\alpha \beta}^{\prime(i)} \varphi_{i}^{\mathrm{T}}\left(x^{\prime}\right) \epsilon \psi_{\beta L}\left(x^{\prime}\right) \\
& + \text { h.c. }
\end{aligned}
$$

Here we have

$$
\tilde{C}^{\prime(i)}=U_{R}^{(\chi) \mathrm{T}} C^{\prime(j) *} U_{L}^{(\psi) *} W_{j i}^{*} .
$$

\subsection{Invariant couplings for two lepton families}

Now we impose our principle of maximal CP invariance on the Yukawa interaction (67). We want to find out what this implies for the coupling matrices $C_{l}^{(j)}, C_{q}^{\prime(j)}$ and $C_{q}^{(j)}$. We start by considering only the leptonic part of $\mathscr{L}_{\text {Yuk }}$ in (67),

$$
\begin{aligned}
\mathscr{L}_{\mathrm{Yuk}, l}(x)= & -\bar{l}_{\alpha R}(x) C_{l \alpha \beta}^{(j)} \varphi_{j}^{\dagger}(x)\left(\begin{array}{c}
\nu_{\beta L}(x) \\
l_{\beta L}(x)
\end{array}\right) \\
& + \text { h.c. }
\end{aligned}
$$

As explained in section 4.2 we can, without loss of generality, suppose (68) to hold. Now we consider a generic $\mathrm{CP}_{g}$ transformation for which (54) holds for the Higgs fields. This $\mathrm{CP}_{g}$ can be extended to an invariance of $\mathscr{L}_{\text {Yuk }, l}(x)$ if and only if we can find $U(2)$ matrices $U_{R}^{(l)}$ and $U_{L}^{(l)}$ in (73) such that, according to (86), we have

$$
U_{R}^{(l) \mathrm{T}} C_{l}^{(j) *} U_{L}^{(l) *} W_{j i}=C_{l}^{(i)} .
$$

For the principle of maximal CP invariance to hold we must be able to find matrices $U_{R}^{(l)}, U_{L}^{(l)}$ solving (91) for all four transformations $\mathrm{CP}_{g}^{(i)}, \mathrm{CP}_{g, 1}^{(i i)}, \mathrm{CP}_{g, 2}^{(i i)}$ and $\mathrm{CP}_{g, 3}^{(i i)}$, with the corresponding $W_{j i}$ from Tab. I.

Let us first consider the case

$$
c_{l 2}^{(1)}>0, \quad c_{l 3}^{(1)}>0, \quad c_{l 2}^{(1)} \neq c_{l 3}^{(1)} .
$$


This corresponds to non-vanishing and unequal masses for the leptons $l_{2}$ and $l_{3}$ after EWSB. As we show in appendix B we have, if (92) holds, solutions of (91) for all four $\mathrm{CP}_{g}$ transformations only if the matrix $C_{l}^{(2)}$ has the following structure:

$$
C_{l}^{(2)}=\left(\begin{array}{cc}
0 & C_{l 23}^{(2)} \\
C_{l 32}^{(2)} & 0
\end{array}\right)
$$

The possible values for $C_{l 23}^{(2)}$ and $C_{l 32}^{(2)}$ are listed in Tab. III (see (B.48), (B.49), (B.57) and (B.58)).

\begin{tabular}{|c|c|}
\hline$C_{l 23}^{(2)}$ & $C_{l 32}^{(2)}$ \\
\hline \hline$c_{l 3}^{(1)}$ & $c_{l 2}^{(1)}$ \\
$c_{l 3}^{(1)}$ & $-c_{l 2}^{(1)}$ \\
$c_{l 2}^{(1)}$ & $c_{l 3}^{(1)}$ \\
$c_{l 2}^{(1)}$ & $-c_{l 3}^{(1)}$ \\
\hline
\end{tabular}

TABLE III: The possible values for $C_{l 23}^{(2)}$ and $C_{l 32}^{(2)}$ for the case that (92) holds.

The corresponding matrices $U_{R, L}^{(l)}$ for all four $\mathrm{CP}_{g}$ symmetries are given in appendix $B$ To see the physical consequences of this result we look at $\mathscr{L}_{\text {Yuk }, l}$ after EWSB. Inserting for the Higgs fields the physical expressions (37) and (38) we get from (90)

$$
\begin{aligned}
& \mathscr{L}_{\mathrm{Yuk}, l}= \\
&-c_{l 2}^{(1)} \frac{1}{\sqrt{2}}\left(v_{0}+\rho^{\prime}(x)\right) \bar{l}_{2}(x) l_{2}(x) \\
&-c_{l 3}^{(1)} \frac{1}{\sqrt{2}}\left(v_{0}+\rho^{\prime}(x)\right) \bar{l}_{3}(x) l_{3}(x) \\
&-H^{-}(x)\left[C_{l 23}^{(2)} \bar{l}_{2}(x) \omega_{L} \nu_{3}(x)\right. \\
&\left.+C_{l 32}^{(2)} \bar{l}_{3}(x) \omega_{L} \nu_{2}(x)\right] \\
&-H^{+}(x)\left[C_{l 23}^{(2)} \bar{\nu}_{3}(x) \omega_{R} l_{2}(x)\right. \\
&\left.+C_{l 32}^{(2)} \bar{\nu}_{2}(x) \omega_{R} l_{3}(x)\right] \\
&-\frac{h^{\prime}(x)}{\sqrt{2}}\left\{\bar{l}_{2}(x)\left[C_{l 23}^{(2)} \omega_{L}+C_{l 32}^{(2)} \omega_{R}\right] l_{3}(x)\right. \\
&\left.+\bar{l}_{3}(x)\left[C_{l 23}^{(2)} \omega_{R}+C_{l 32}^{(2)} \omega_{L}\right] l_{2}(x)\right\} \\
&-\frac{i h^{\prime \prime}(x)}{\sqrt{2}}\left\{\bar{l}_{2}(x)\left[-C_{l 23}^{(2)} \omega_{L}+C_{l 32}^{(2)} \omega_{R}\right] l_{3}(x)\right. \\
&\left.+\bar{l}_{3}(x)\left[C_{l 23}^{(2)} \omega_{R}-C_{l 32}^{(2)} \omega_{L}\right] l_{2}(x)\right\}
\end{aligned}
$$

with the chirality projectors

$$
\omega_{R}:=\frac{1+\gamma_{5}}{2} \quad \text { and } \quad \omega_{L}:=\frac{1-\gamma_{5}}{2} .
$$

Here we can read off the lepton masses

$$
\begin{aligned}
& m_{l 2}=c_{l 2}^{(1)} \frac{v_{0}}{\sqrt{2}}, \\
& m_{l 3}=c_{l 3}^{(1)} \frac{v_{0}}{\sqrt{2}} .
\end{aligned}
$$

Identifying the lepton 3 with the $\tau$ lepton we see that in all cases listed in Tab. [II] either $\left|C_{l 23}^{(2)}\right|=m_{\tau} \sqrt{2} / v_{0}$ or $\left|C_{l 32}^{(2)}\right|=m_{\tau} \sqrt{2} / v_{0}$. Thus (94) always contains large lepton flavour-changing neutral currents, FCNCs. These would allow for processes like

$$
l_{2}+l_{2} \rightarrow l_{3}+l_{3}
$$

through diagrams like in Fig. 1. A direct study of process

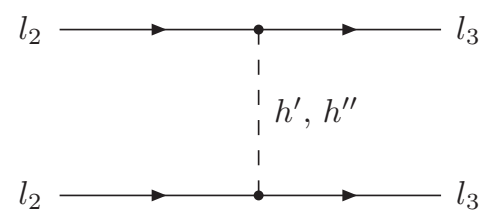

FIG. 1: Two Feynman diagrams for the large FCNC process $l_{2}+l_{2} \rightarrow l_{3}+l_{3}$ reflecting the last two contributions in the Lagrangian (94).

(97), $\mu^{-}+\mu^{-} \rightarrow \tau^{-}+\tau^{-}$, would be a topic for a muon collider which, however, is far in the future. But the couplings in Fig. 1 would also lead to spectacular leptonflavour-violating events in deep inelastic muon-nucleon scattering,

$$
\mu^{-}+N \rightarrow \mu^{+}+\tau^{-}+\tau^{-}+X .
$$

Two of the corresponding tree level Feynman diagrams are shown in Fig. 2, Here $X$ stands for the hadronic

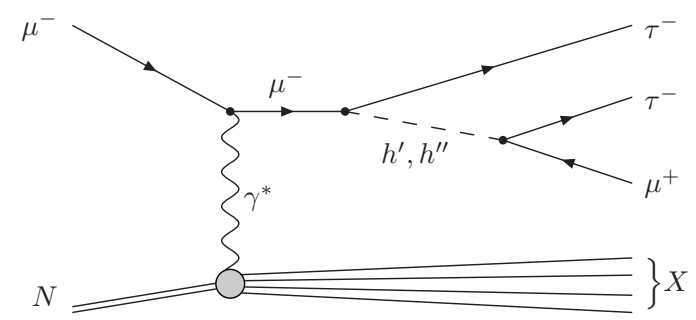

FIG. 2: Two Feynman diagrams for the deep inelastic muonnucleon scattering process which would reveal FCNCs corresponding to the couplings in Fig. 1

final state. Since such FCNCs were never observed we consider them to be unacceptable phenomenologically.

The next case to study is

$$
c_{l 2}^{(1)}=c_{l 3}^{(1)}>0 .
$$

There we can construct a coupling (90) satisfying the principle of maximal $\mathrm{CP}$ invariance and having no 
FCNCs. We give the details in appendix B. However, here we have, according to (96) equal lepton masses, $m_{l 2}=m_{l 3}$, which is, again, unacceptable phenomenologically.

It remains to be seen what happens for the case of one massless and one massive lepton. Taking, by convention, $l_{3}$ to be the massive lepton we have the case

$$
c_{l 2}^{(1)}=0, \quad c_{l 3}^{(1)}>0 .
$$

Here we shall prescribe the form of the matrices $U_{R}^{(l)}$ and $U_{L}^{(l)}$ for the four $\mathrm{CP}_{g}$ transformations as shown in Tab. IV $\mathrm{CP}_{g, 2}^{(i i)}$ is the standard CP transformation for all fields, $\mathrm{CP}_{g, 2}^{(i i)}=\mathrm{CP}_{s}$. We require now invariance of the Yukawa interaction (90) under these four $\mathrm{CP}_{g}$ transformations, that is, we require (91) to hold with the corresponding $W_{i j}$ from Tab. [V] It is easy to check that starting with $c_{l 2}^{(1)} \geq 0, c_{l 3}^{(1)}>0$ instead of (100) these invariances require $c_{l 2}^{(1)}=0$ and

$$
C_{l}^{(2)}=\left(\begin{array}{cc}
-c_{l 3}^{(1)} & 0 \\
0 & 0
\end{array}\right)
$$

see (B.110). The resulting Yukawa term reads

$$
\begin{aligned}
\mathscr{L}_{\text {Yuk }, l}(x)=-c_{l 3}^{(1)} & \left\{\bar{l}_{3 R}(x) \varphi_{1}^{\dagger}(x)\left(\begin{array}{c}
\nu_{3 L}(x) \\
l_{3 L}(x)
\end{array}\right)\right. \\
& \left.-\bar{l}_{2 R}(x) \varphi_{2}^{\dagger}(x)\left(\begin{array}{c}
\nu_{2 L}(x) \\
l_{2 L}(x)
\end{array}\right)\right\}+ \text { h.c. }
\end{aligned}
$$

Note the high degree of symmetry between the families here. However, after EWSB we get, inserting (37) and (38) for the Higgs fields and using (96),

$$
\begin{aligned}
\mathscr{L}_{\text {Yuk }, l}(x)= & -m_{l 3}\left(1+\frac{\rho^{\prime}(x)}{v_{0}}\right) \bar{l}_{3}(x) l_{3}(x) \\
& +\frac{m_{l 3}}{v_{0}} h^{\prime}(x) \bar{l}_{2}(x) l_{2}(x) \\
& +i \frac{m_{l 3}}{v_{0}} h^{\prime \prime}(x) \bar{l}_{2}(x) \gamma_{5} l_{2}(x) \\
& +\frac{\sqrt{2} m_{l 3}}{v_{0}}\left[H^{+}(x) \bar{\nu}_{2}(x) \omega_{R} l_{2}(x)\right. \\
& \left.+H^{-}(x) \bar{l}_{2}(x) \omega_{L} \nu_{2}(x)\right] .
\end{aligned}
$$

The lepton $l_{3}$ has become massive and couples to the physical $\rho^{\prime}$ Higgs. The lepton $l_{2}$ is massless but couples to $h^{\prime}, h^{\prime \prime}$ and the charged Higgs bosons $H^{ \pm}$.

In appendix B we give a general discussion of the case where (100) holds; that is, where lepton $l_{2}$ is massless and lepton $l_{3}$ massive. We show there that the requirements of maximal CP invariance plus absence of FCNCs uniquely leads to the coupling (102).

\begin{tabular}{|l|c|c|c|}
\hline $\mathrm{CP}_{g}$ & $\mathrm{~W}$ & $U_{R}^{(l)}$ & $U_{L}^{(l)}$ \\
\hline \hline $\mathrm{CP}_{g}^{(i)}$ & $\epsilon$ & $\epsilon$ & $\sigma^{1}$ \\
$\mathrm{CP}_{g, 1}^{(i i)}$ & $\sigma^{3}$ & $-\sigma^{3}$ & $\mathbb{1}_{2}$ \\
$\mathrm{CP}_{g, 2}^{(i i)}$ & $\mathbb{1}_{2}$ & $\mathbb{1}_{2}$ & $\mathbb{1}_{2}$ \\
$\mathrm{CP}_{g, 3}^{(i i)}$ & $\sigma^{1}$ & $-\sigma^{1}$ & $\sigma^{1}$ \\
\hline
\end{tabular}

TABLE IV: The matrices $W, U_{R}^{(l)}$ and $U_{L}^{(l)}$ for the four $\mathrm{CP}_{g}$ transformations for the case of one massless and one massive lepton; see (100).

\subsection{Invariant couplings for two quark families}

In this section we study the quark part of the Lagrangian (67). Let us first look at the term which generates masses for the $u$-type quarks,

$$
\begin{aligned}
\mathscr{L}_{\mathrm{Yuk}, q^{\prime}}(x)= & \bar{u}_{\alpha R}(x) C_{q \alpha \beta}^{\prime(j)} \varphi_{j}^{\mathrm{T}}(x) \epsilon\left(\begin{array}{l}
u_{\beta L}(x) \\
d_{\beta L}^{\prime}(x)
\end{array}\right) \\
& + \text { h.c. }
\end{aligned}
$$

Here we can suppose without loss of generality that $C_{q}^{\prime(1)}$ is as in (69). As for the case of the leptons in section 4.3 we ask if $\mathscr{L}_{\text {Yuk }, q^{\prime}}$ in (104) allows for the implementation of our principle of maximal CP invariance. That is, we ask for matrices $U_{R}^{(u)}, U_{L}^{(u)}$ in (73) which satisfy either (78) or (79) and allow us to solve (see (89))

$$
U_{R}^{(u) \mathrm{T}} C_{q}^{(j) *} U_{L}^{(u) *} W_{j i}^{*}=C_{q}^{(i)},
$$

for all four CP symmetries with $W_{j i}$ as given in Tab. [I] Since all $W$ matrices are real (105) is completely analogous to (91). We can immediately conclude from the results of section 4.3 that for the case

$$
c_{u 2}^{(1)}>0, \quad c_{u 3}^{(1)}>0, \quad c_{u 2}^{(1)} \neq c_{u 3}^{(1)}
$$

the principle of maximal $\mathrm{CP}$ invariance leads to large FCNCs. Here it is important to note that these FCNCs are generated for the physical mass eigenfields $u_{2}$ and $u_{3}$. Requiring the absence of these FCNCs then allows for only two possibilities for a non-zero coupling $\mathscr{L}_{\text {Yuk }, q^{\prime}}$. Either we must have non-zero equal masses for the quarks $u_{2}$ and $u_{3}$ or we must have $u_{2}$ massless and $u_{3}$ massive. Discarding the former for phenomenological reasons we are left with the case of a massless $u_{2}=c$ quark and a massive $u_{3}=t$ quark. Now we prescribe the matrices $U_{R}^{(u)}$ and $U_{L}^{(u)}$ for the four $\mathrm{CP}_{g}$ transformations as for the lepton case in Tab. IV. That is, we set for all $\mathrm{CP}_{g}$ transformations

$$
\begin{aligned}
& U_{R}^{(u)}=U_{R}^{(l)}, \\
& U_{L}^{(u)}=U_{L}^{(l)} .
\end{aligned}
$$


With (107) we find that the matrices $C_{q}^{\prime(j)}(j=1,2)$ have to be as follows:

$$
\begin{aligned}
& C_{q}^{(1)}=\left(\begin{array}{cc}
0 & 0 \\
0 & c_{u 3}^{(1)}
\end{array}\right), \quad c_{u 3}^{(1)}>0 \\
& C_{q}^{(2)}=\left(\begin{array}{cc}
-c_{u 3}^{(1)} & 0 \\
0 & 0
\end{array}\right) .
\end{aligned}
$$

The resulting coupling term (104) reads

$$
\begin{aligned}
\mathscr{L}_{\mathrm{Yuk}, q^{\prime}}(x)=c_{u 3}^{(1)} & \left\{\bar{u}_{3 R}(x) \varphi_{1}^{\mathrm{T}}(x) \epsilon\left(\begin{array}{l}
u_{3 L}(x) \\
d_{3 L}^{\prime}(x)
\end{array}\right)\right. \\
& \left.-\bar{u}_{2 R}(x) \varphi_{2}^{\mathrm{T}}(x) \epsilon\left(\begin{array}{l}
u_{2 L}(x) \\
d_{2 L}^{\prime}(x)
\end{array}\right)\right\}+ \text { h.c. }
\end{aligned}
$$

As for the case of the leptons (see appendix B) we can show the following. For $c_{u 2}^{(1)}=0, c_{u 3}^{(1)}>0$ the principle of maximal $\mathrm{CP}$ invariance together with the requirement of absence of FCNCs leads uniquely to the coupling (109).

We turn next to the term in (67) which generates masses for $d$-type quarks

$$
\begin{aligned}
\mathscr{L}_{\text {Yuk }, q}(x)= & -\bar{d}_{\alpha R}^{\prime}(x) C_{q \alpha \beta}^{(j)} \varphi_{j}^{\dagger}(x)\left(\begin{array}{l}
u_{\beta L}(x) \\
d_{\beta L}^{\prime}(x)
\end{array}\right) \\
& + \text { h.c. }
\end{aligned}
$$

Here the standard form for $C_{q}^{(1)}$ is given in (70) and (71). Note that $d_{\beta}^{\prime}$ are - in general - not the mass eigenfields. We shall change to the basis of $d_{\alpha}$ mass eigenfields and the corresponding isospin partners of $u_{\alpha}^{\prime}$ fields according to (72) setting

$$
\begin{aligned}
d_{\alpha R}(x) & =V_{\alpha \beta}^{\dagger} d_{\beta R}^{\prime}, \\
\left(\begin{array}{c}
u_{\alpha L}^{\prime}(x) \\
d_{\alpha L}(x)
\end{array}\right) & =V_{\alpha \beta}^{\dagger}\left(\begin{array}{l}
u_{\beta L}(x) \\
d_{\beta L}^{\prime}(x)
\end{array}\right) .
\end{aligned}
$$

The coupling term (110) reads now

$$
\begin{aligned}
\mathscr{L}_{\text {Yuk }, q}(x)= & -\bar{d}_{\alpha R}(x) \tilde{C}_{q \alpha \beta}^{(j)} \varphi_{j}^{\dagger}(x)\left(\begin{array}{l}
u_{\beta L}^{\prime}(x) \\
d_{\beta L}(x)
\end{array}\right) \\
& + \text { h.c. }
\end{aligned}
$$

where

$$
\tilde{C}_{q}^{(j)}=V^{\dagger} C_{q}^{(j)} V, \quad j=1,2 .
$$

From (70) we see that this implies

$$
\tilde{C}_{q}^{(1)}=\left(\begin{array}{cc}
c_{d 2}^{(1)} & 0 \\
0 & c_{d 3}^{(1)}
\end{array}\right) .
$$

Now we can proceed as for the lepton case. We see that requiring the principle of maximal $\mathrm{CP}$ invariance leads for the case

$$
c_{d 2}^{(1)}>0, \quad c_{d 3}^{(1)}>0, \quad c_{d 2}^{(1)} \neq c_{d 3}^{(1)}
$$

to large FCNCs among the physical $d$-quark mass eigenfields. These FCNCs can only be avoided if we require either equal masses $m_{d 2}=m_{d 3}$ or $m_{d 2}=0$ and $m_{d 3} \neq 0$. Again we discard the former possibility for phenomenological reasons. For the case $m_{d 2}=0, m_{d 3} \neq 0$ we shall again prescribe the $\mathrm{CP}_{g}$ transformation matrices of the fermion fields. But we have to remember that we have already prescribed the transformation matrices $U_{L}^{(u)}$ for the left-handed quark doublets in (107). Since the same doublets appear in (110) we can here only prescribe $U_{R}^{(d)}$ in (73), since everything else is already fixed. Note that $U_{R}^{(d)}$ refers again to the $d_{\alpha}^{\prime}$ fields. We prescribe here

$$
U_{R}^{(d)}=U_{R}^{(l)}
$$

as in Tab. IV. This leads to

$$
V=\mathbb{1}_{2}
$$

that is, to a CKM matrix equal to unity in the $2-3$ sector as we show in appendix C. The final form of the coupling term $\mathscr{L}_{\text {Yuk }, q}$ is as follows:

$$
\begin{aligned}
\mathscr{L}_{\mathrm{Yuk}, q}(x)=-c_{d 3}^{(1)} & \left\{\bar{d}_{3 R}(x) \varphi_{1}^{\dagger}(x)\left(\begin{array}{l}
u_{3 L}(x) \\
d_{3 L}(x)
\end{array}\right)\right. \\
& \left.-\bar{d}_{2 R}(x) \varphi_{2}^{\dagger}(x)\left(\begin{array}{l}
u_{2 L}(x) \\
d_{2 L}(x)
\end{array}\right)\right\}+ \text { h.c. }
\end{aligned}
$$

Note that with (117) we have $d_{\alpha R, L}^{\prime}=d_{\alpha R, L}$ for the $d$ type fields.

In appendix $\mathrm{C}$ we give a general discussion of the case $c_{d 2}^{(1)}=0, c_{d 3}^{(1)}>0$, that is of the case where $d_{2}$ is massless and $d_{3}$ massive.

\section{DISCUSSION}

In this section we collect the results found in the previous sections and subsequently discuss their physical consequences. We have investigated a two-Higgs-doublet model having four generalised CP transformations as symmetries. We have introduced the principle of maximal CP invariance which requires that these four symmetries are extendable to the full Lagrangian.

In sections 2 and 3 we have discussed the Higgs sector of the model which is characterised by the requirement of $\mathrm{CP}_{g}^{(i)}$ invariance. We have seen that this leads automatically to three more $\mathrm{CP}_{g}$ invariances, $\mathrm{CP}_{g, 1}^{(i i)}, \mathrm{CP}_{g, 2}^{(i i)}$ and $\mathrm{CP}_{g, 3}^{(i i)}$. The EWSB breaks $\mathrm{CP}_{g}^{(i)}$ and $\mathrm{CP}_{g, 3}^{(i i)}$ spontaneously. At tree level, which we have discussed in this paper, the symmetries $\mathrm{CP}_{g, 1}^{(i i)}$ and $\mathrm{CP}_{g, 2}^{(i i)}$ are unbroken.

In section 4 we studied if we can implement the principle of maximal CP invariance, that is, if our four $\mathrm{CP}_{g}$ symmetries can be extended to the coupling of the fermions to the Higgs fields. For this we introduced the 
fermion families; see Tab. II taking the neutrinos as massless. We found in section 4.1 that requiring a single family to have a $\mathrm{CP}_{g}^{(i)}$ invariant coupling leads necessarily to the coupling being identically zero. Thus, we have the interesting conclusion that a single fermion family with non-zero couplings and, therefore, masses is not consistent with $\mathrm{CP}_{g}^{(i)}$ invariance, and therefore, a forteriori, with the principle of maximal $\mathrm{CP}$ invariance.

In sections 4.2 to 4.4 we discussed non-zero couplings of two families to the Higgs doublets, always requiring the principle of maximal CP invariance. We took the two families to be the second and the third. We found that unequal non-zero masses for the leptons $l_{2}$ and $l_{3}$, the quarks $u_{2}$ and $u_{3}$, as well as $d_{2}$ and $d_{3}$ always implied large flavour-changing neutral currents (FCNCs). The absence of large FCNCs required either equal masses of corresponding fermions $\left(m_{l 2}=m_{l 3}\right.$ etc. $)$ or one fermion massless, the other massive. Discarding the equal mass case on phenomenological grounds we were, thus, left with the possibility

$$
\begin{aligned}
m_{l 2} & =0, & & m_{l 3} \neq 0, \\
m_{u 2} & =0, & & m_{u 3} \neq 0, \\
m_{d 2} & =0, & & m_{d 3} \neq 0 .
\end{aligned}
$$

The specific set of CP symmetries defined by (73) together with Tab. IV] (107) and (116) guarantees the absence of large FCNCs and requires vanishing masses for family 2. As a further consequence the CKM matrix between the families 2 and 3 has to be equal to unity; see (117). Combining (102), (109) and (118) we find for the full Yukawa part of the Lagrangian the simple form

$$
\begin{aligned}
\mathscr{L}_{\text {Yuk }}(x)=-c_{l 3}^{(1)} & \left\{\bar{l}_{3 R}(x) \varphi_{1}^{\dagger}(x)\left(\begin{array}{c}
\nu_{3 L}(x) \\
l_{3 L}(x)
\end{array}\right)\right. \\
& \left.-\bar{l}_{2 R}(x) \varphi_{2}^{\dagger}(x)\left(\begin{array}{c}
\nu_{2 L}(x) \\
l_{2 L}(x)
\end{array}\right)\right\} \\
+c_{u 3}^{(1)} & \left\{\bar{u}_{3 R}(x) \varphi_{1}^{\mathrm{T}}(x) \epsilon\left(\begin{array}{l}
u_{3 L}(x) \\
d_{3 L}(x)
\end{array}\right)\right. \\
& \left.-\bar{u}_{2 R}(x) \varphi_{2}^{\mathrm{T}}(x) \epsilon\left(\begin{array}{c}
u_{2 L}(x) \\
d_{2 L}(x)
\end{array}\right)\right\} \\
-c_{d 3}^{(1)} & \left\{\bar{d}_{3 R}(x) \varphi_{1}^{\dagger}(x)\left(\begin{array}{c}
u_{3 L}(x) \\
d_{3 L}(x)
\end{array}\right)\right. \\
& \left.-\bar{d}_{2 R}(x) \varphi_{2}^{\dagger}(x)\left(\begin{array}{c}
u_{2 L}(x) \\
d_{2 L}(x)
\end{array}\right)\right\}+ \text { h.c. }
\end{aligned}
$$

In this model the first family remains uncoupled to the Higgs fields.

For the convenience of the reader we summarise here the generalised CP symmetries of the full Lagrangian (2) with three generations of fermions, the Higgs part given by (3) and (27), and the Yukawa term given by (120).
For any $\mathrm{CP}_{g}$ we transform the gauge bosons as in (15) and the first generation fermions as in (61) where $\xi_{1}$ to $\xi_{5}$ remain arbitrary. The Higgs fields are transformed according to (54) and the second and third generation fermions according to (73). The matrices $W$ in (54) and $U_{L}^{(l)}$ to $U_{R}^{(d)}$ in (73) are summarised in Tab. V. In appendix D we discuss the relation of these $\mathrm{CP}_{g}$ invariances to conventional discrete symmetries.

After EWSB we insert the Higgs fields parametrised by the physical fields as in (37) and (38) and use the relations

$$
\begin{aligned}
& m_{l 3}=c_{l 3}^{(1)} \frac{v_{0}}{\sqrt{2}} \equiv m_{\tau}, \\
& m_{u 3}=c_{u 3}^{(1)} \frac{v_{0}}{\sqrt{2}} \equiv m_{t}, \\
& m_{d 3}=c_{d 3}^{(1)} \frac{v_{0}}{\sqrt{2}} \equiv m_{b} .
\end{aligned}
$$

We find then from (120) with $\omega_{R, L}$ defined in (95)

$$
\begin{aligned}
& \mathscr{L}_{\text {Yuk }}(x)= \\
& -m_{l 3}\left(1+\frac{\rho^{\prime}(x)}{v_{0}}\right) \bar{l}_{3}(x) l_{3}(x) \\
& -m_{u 3}\left(1+\frac{\rho^{\prime}(x)}{v_{0}}\right) \bar{u}_{3}(x) u_{3}(x) \\
& -m_{d 3}\left(1+\frac{\rho^{\prime}(x)}{v_{0}}\right) \bar{d}_{3}(x) d_{3}(x) \\
& +\frac{h^{\prime}(x)}{v_{0}} \quad\left[m_{l 3} \bar{l}_{2}(x) l_{2}(x)\right. \\
& +m_{u 3} \bar{u}_{2}(x) u_{2}(x) \\
& \left.+m_{d 3} \bar{d}_{2}(x) d_{2}(x)\right] \\
& +i \frac{h^{\prime \prime}(x)}{v_{0}}\left[m_{l 3} \bar{l}_{2}(x) \gamma_{5} l_{2}(x)\right. \\
& -m_{u 3} \bar{u}_{2}(x) \gamma_{5} u_{2}(x) \\
& \left.+m_{d 3} \bar{d}_{2}(x) \gamma_{5} d_{2}(x)\right] \\
& +\left\{\frac { H ^ { + } ( x ) \sqrt { 2 } } { v _ { 0 } } \left[m_{l 3} \bar{\nu}_{2}(x) \omega_{R} l_{2}(x)\right.\right. \\
& \text { - } m_{u 3} \bar{u}_{2}(x) \omega_{L} d_{2}(x) \\
& \left.\left.+m_{d 3} \bar{u}_{2}(x) \omega_{R} d_{2}(x)\right]+ \text { h.c. }\right\} \text {. }
\end{aligned}
$$

We discuss now the transformation properties of the physical fields after EWSB under the $\mathrm{CP}_{g}$ transformations of Tab. V] The symmetries $\mathrm{CP}_{g}^{(i)}$ and $\mathrm{CP}_{g, 3}^{(i)}$ are spontaneously broken. Thus they are not explicitly visible for the physical fields. The symmetries $\mathrm{CP}_{g, 1}^{(i i)}$ and $\mathrm{CP}_{g, 2}^{(i i)}$ are unbroken and are thus directly reflected by the physical fields. The transformation of the gauge bosons is always given by (15); see the remark after (21). The 
transformations of the physical Higgs fields under $\mathrm{CP}_{g, 1}^{(i i)}$ and $\mathrm{CP}_{g, 2}^{(i i)}$ are given in (58) and (59), respectively. For the first generation fermions both, $\mathrm{CP}_{g, 1}^{(i i)}$ and $\mathrm{CP}_{g, 2}^{(i i)}$, can be taken to be the standard CP transformation, setting $\xi_{1}=\ldots=\xi_{5}=0$ in (61). The transformation $\mathrm{CP}_{g, 2}^{(i i)}$ acts as standard CP transformation also for the second and third fermion families; see Tab. V. The transformation $\mathrm{CP}_{g, 1}^{(i i)}$ acts, according to Tab. $\mathrm{V}$ on the second and third generation lepton fields as follows; see (73):

$$
\begin{aligned}
\mathrm{CP}_{g, 1}^{(i i)}: \nu_{\mu L}(x) & \rightarrow \gamma^{0} S(C) \bar{\nu}_{\mu L}^{\mathrm{T}}\left(x^{\prime}\right), \\
\mu_{L}(x) & \rightarrow \gamma^{0} S(C) \bar{\mu}_{L}^{\mathrm{T}}\left(x^{\prime}\right), \\
\mu_{R}(x) & \rightarrow-\gamma^{0} S(C) \bar{\mu}_{R}^{\mathrm{T}}\left(x^{\prime}\right), \\
\nu_{\tau L}(x) & \rightarrow \gamma^{0} S(C) \bar{\nu}_{\tau L}^{\mathrm{T}}\left(x^{\prime}\right), \\
\tau(x) & \rightarrow \gamma^{0} S(C) \bar{\tau}^{\mathrm{T}}\left(x^{\prime}\right) .
\end{aligned}
$$

Thus the $\mathrm{CP}_{g, 1}^{(i i)}$ transformations for the states of $\nu_{\mu}, \nu_{\tau}$ and $\tau$ are as for the standard CP transformation. For the muons, however, we have at the level of the states

$$
\begin{aligned}
& \mathrm{CP}_{g, 1}^{(i i)}:\left|\mu^{-}(\mathbf{k}, s)\right\rangle \rightarrow(-1)^{s+1 / 2}\left|\mu^{+}(-\mathbf{k},-s)\right\rangle, \\
& \mathrm{CP}_{g, 2}^{(i i)}:\left|\mu^{-}(\mathbf{k}, s)\right\rangle \rightarrow\left|\mu^{+}(-\mathbf{k},-s)\right\rangle .
\end{aligned}
$$

Here $\mathbf{k}$ is the momentum and $s= \pm 1 / 2$ is the helicity of the state. Thus $\mathrm{CP}_{g, 1}^{(i i)}$ differs from the standard $\mathrm{CP}$ transformation $\mathrm{CP}_{g, 2}^{(i i)}$ by an extra minus sign in the transformation of the right-handed $\mu^{-}(s=1 / 2)$. Note that in our theory as it has been developed so far the muon is massless. Thus its helicity is a Lorentz-invariant quantity. For the second and third generation quarks the transformations $\mathrm{CP}_{g, 1}^{(i i)}$ and $\mathrm{CP}_{g, 2}^{(i i)}$ act analogously to the lepton case.

Finally we stress again that the theory defined - before EWSB - by the Lagrangian (2) with $\mathscr{L}_{\varphi}$ given by (3) and (27)-(29) and $\mathscr{L}_{\text {Yuk }}$ given by (120) is symmetric under all four $\mathrm{CP}_{g}$ transformations as defined in Tab. V Moreover, it is the most general theory with these symmetries. That is, there is no further symmetric renormalisable term which could be added.

We consider it noteworthy that our symmetry principles require more than one family. For two families we get in a natural way mass hierarchies. Choosing the simplest extension to three families we get masses unequal to zero only for $\tau, t$ and $b$ whereas all other leptons and quarks, $\mu, e, c, u, s, d$ stay massless. In addition, the CKM matrix of the quarks equals the unit matrix, $V=\mathbb{1}$. Clearly, all this is not quite as one observes it in Nature. On the other hand, as a first approximation, it is also not so

\begin{tabular}{|c|c|c|c|}
\hline $\mathrm{CP}_{g}$ & $W$ & $U_{R}^{(l)}=U_{R}^{(u)}=U_{R}^{(d)}$ & $U_{L}^{(l)}=U_{L}^{(u)}$ \\
\hline \hline $\mathrm{CP}_{g}^{(i)}$ & $\epsilon$ & $\epsilon$ & $\sigma^{1}$ \\
$\mathrm{CP}_{g, 1}^{(i i)}$ & $\sigma^{3}$ & $-\sigma^{3}$ & $\mathbb{1}_{2}$ \\
$\mathrm{CP}_{g, 2}^{(i i)}$ & $\mathbb{1}_{2}$ & $\mathbb{1}_{2}$ & $\mathbb{1}_{2}$ \\
$\mathrm{CP}_{g, 3}^{(i i)}$ & $\sigma^{1}$ & $-\sigma^{1}$ & $\sigma^{1}$ \\
\hline
\end{tabular}

TABLE V: The matrices $W$ (54) and $U_{L}^{(l)}$ to $U_{R}^{(d)}$ in (73) giving the four $\mathrm{CP}_{g}$ invariances of the Lagrangian with the Yukawa term (120).

bad. We have [18, 19, 20]

$$
\begin{array}{ll}
\frac{m_{e}}{m_{\tau}} \approx 0.00029, & \frac{m_{\mu}}{m_{\tau}} \approx 0.059, \\
\left.\frac{m_{u}}{m_{t}}\right|_{v_{0}} \approx 9.9 \cdot 10^{-6}, & \left.\frac{m_{c}}{m_{t}}\right|_{v_{0}} \approx 0.0036, \\
\left.\frac{m_{d}}{m_{b}}\right|_{v_{0}} \approx 0.0010, & \left.\frac{m_{s}}{m_{b}}\right|_{v_{0}} \approx 0.018 .
\end{array}
$$

Here we have used for the quarks the $\overline{M S}$ masses at the renormalisation point $\mu=v_{0} \approx 246 \mathrm{GeV}$ and $\alpha_{s}\left(m_{Z}\right)=0.119$. This electroweak scale seems to us a natural choice for our purpose. Also the CKM matrix is in Nature not too far from unity. Indeed, one finds for the absolute values $\left|V_{i j}\right|[18]$

$$
\left(\begin{array}{lll}
\left|V_{11}\right| & \left|V_{12}\right| & \left|V_{13}\right| \\
\left|V_{21}\right| & \left|V_{22}\right| & \left|V_{23}\right| \\
\left|V_{31}\right| & \left|V_{32}\right| & \left|V_{33}\right|
\end{array}\right) \approx\left(\begin{array}{lll}
0.974 & 0.227 & 0.004 \\
0.227 & 0.973 & 0.042 \\
0.008 & 0.042 & 0.999
\end{array}\right)
$$

Note that in the $2-3$ sector $V$ is very close to the unit matrix. But clearly a good theory should be able to explain the experimental numbers in (125) and (126) in more detail.

\section{CONCLUSIONS}

We have studied a two-Higgs-doublet model where the scalar sector has four generalised CP symmetries. Two of these symmetries are spontaneously broken by the electroweak symmetry breaking (EWSB). We have introduced the principle of maximal CP invariance requiring that these four CP symmetries can be extended to the full Lagrangian of the theory. We find that for a single fermion family this principle forbids a non-zero fermionHiggs coupling. Thus, if we want massive fermions which arise from non-zero Yukawa couplings we need family replication. We have studied then in detail theories with two fermion families. Here, indeed, we can extend all four CP symmetries to the full Lagrangian with non-zero Yukawa couplings which are, however, highly constrained. Discarding extensions which enforce large flavour-changing neutral currents, we are left with the possibilities of either equal masses for the corresponding 
fermions in the families or large mass hierarchies. Choosing the latter possibility we arrive at a theory with a high degree of symmetry between the two families and absence of flavour-changing neutral currents. The Yukawa part of this theory is given in (120) and after EWSB in (122). Through EWSB one family becomes massive the other stays massless at the tree level, which we have discussed in this paper. We have shown that we can also obtain this theory directly from a symmetry requirement. For this we prescribe the form of the four CP transformations for the lepton and quark fields as shown in Tab. V] Our principle of maximal CP invariance leads then directly to the Yukawa coupling (120) implying one massive and one massless family as well as absence of large FCNCs. Adding a fermion family uncoupled to the Higgs particles we arrive at a model which looks like giving a rough approximation of the structure of fermions observed in Nature. We have massless neutrinos. Concerning the charged fermions we have one massive family which we identify with the third one $(\tau, t, b)$ and two massless ones which we identify with the second $(\mu, c, s)$ and the first $(e, u, d)$ families. In our model the CKM matrix between the quark generations is equal to unity. As for any THDM, the spectrum of physical Higgs particles consists of three neutral scalars, $\rho^{\prime}, h^{\prime}$ and $h^{\prime \prime}$, and the charged Higgs bosons $H^{ \pm}$. The neutral Higgs particle $\rho^{\prime}$ - which has essentially the same properties as the SM Higgs - couples exclusively to the third family of fermions. The other Higgs bosons $h^{\prime}, h^{\prime \prime}$ and $H^{ \pm}$couple exclusively to the second family of fermions. The first fermion family remains uncoupled to the Higgs bosons. Of course, in reality these statements are expected to be only approximately true. Thus, many open problems remain: Suppose that we start from our highly CPsymmetric theory. How can we obtain masses also for the first and second fermion families and the CP violation in the CKM matrix? Can we get the right amount of $\mathrm{CP}$ violation to meet the Sakharov criteria for dynamical generation of the baryon-antibaryon asymmetry in the Universe? What are the effects of radiative corrections in the theory? Can the theory be obtained for instance in some grand unified scenario by integrating out heavy modes? These questions clearly go beyond the scope of the present paper and must be left for further studies. To summarise: we have discussed a two-Higgs-doublet model in which the requirement of maximal CP invariance provides a mechanism to obtain interesting structures for fermion masses and couplings. It remains to be seen if Nature makes use of such a mechanism or if our theory is only a caricature of reality. The experiments at the LHC may be able to tell.

\section{Acknowledgments}

The authors would like to thank R. Barbieri and C. Ewerz for useful discussions and M. Jamin for correspondence concerning the quark mass values.

\section{APPENDIX A: TWO GENERATION MIXING IN CP TRANSFORMATIONS}

In this appendix we discuss the solutions of (77). Every matrix $U \in U(2)$ can be represented as

$$
U=e^{i \xi}\left(\begin{array}{cc}
\alpha & \beta \\
-\beta^{*} & \alpha^{*}
\end{array}\right)
$$

with $\xi$ real and

$$
|\alpha|^{2}+|\beta|^{2}=1
$$

Inserting (A.1) in (77) we get

$$
\begin{aligned}
|\alpha|^{2}-\beta^{2} & =-e^{i \varkappa}, \\
\alpha\left(\beta+\beta^{*}\right) & =0, \\
|\alpha|^{2}-\beta^{* 2} & =-e^{i \varkappa} .
\end{aligned}
$$

It follows that

$$
\begin{aligned}
e^{-i \varkappa} & =e^{i \varkappa}, \\
\Rightarrow \quad e^{i \varkappa} & = \pm 1 .
\end{aligned}
$$

Thus we have two different types of solutions of (77): type (a) where $e^{i \varkappa}=1$ and type (b) where $e^{i \varkappa}=-1$.

For the case (a) we find from (A.3)

$$
\begin{aligned}
|\alpha|^{2}-\beta^{2} & =-1, \\
\beta^{2} & =\beta^{* 2}
\end{aligned}
$$

and together with A.2 we have

$$
|\beta|^{2}+\beta^{2}=2 \text {. }
$$

This gives

$$
\begin{gathered}
\beta^{2}=1, \\
\beta= \pm 1, \\
\alpha=0 .
\end{gathered}
$$

Thus, we get for the case (a) the solution of (77) for $\beta=1$ as

$$
U^{(a)}=e^{i \xi}\left(\begin{array}{cc}
0 & 1 \\
-1 & 0
\end{array}\right)
$$

Taking $\beta=-1$ gives the same after a redefinition of the phase $\xi$. This proves (78).

Turning to the case (b), $e^{i \varkappa}=-1$, we get from A.3

$$
\begin{gathered}
|\alpha|^{2}-\beta^{2}=1, \\
\alpha\left(\beta+\beta^{*}\right)=0 .
\end{gathered}
$$

Together with A.2 we have

$$
\begin{aligned}
\beta^{2} & =-|\beta|^{2}, \\
\beta & = \pm i \beta^{\prime} \quad \text { with } \beta^{\prime} \geq 0 .
\end{aligned}
$$


Defining

$$
\alpha^{\prime}=\mp i \alpha
$$

and inserting in (A.1) we get

$$
\begin{gathered}
U^{(b)}=( \pm i) e^{i \xi}\left(\begin{array}{cc}
\alpha^{\prime} & \beta^{\prime} \\
\beta^{\prime} & -\alpha^{\prime *}
\end{array}\right), \\
\text { with } \beta^{\prime} \geq 0, \quad\left|\alpha^{\prime}\right|^{2}+\beta^{\prime 2}=1 .
\end{gathered}
$$

A redefinition of the phase $\xi$ and the variables $\alpha^{\prime}, \beta^{\prime}$ proves (79).

\section{APPENDIX B: INVARIANT COUPLINGS FOR TWO LEPTON FAMILIES, DETAILS}

In this appendix we discuss the structure of the leptonHiggs coupling (90) requiring invariance under all four transformations $\mathrm{CP}_{g}^{(i)}, \mathrm{CP}_{g, 1}^{(i i)}, \mathrm{CP}_{g, 2}^{(i i)}$ and $\mathrm{CP}_{g, 3}^{(i i)}$. We split the discussion according to the different possibilities for the lepton masses in the following subsections.

\section{The case of different non-vanishing masses}

In this subsection we discuss the structure of the lepton-Higgs coupling requiring invariance under all four transformations $\mathrm{CP}_{g}^{(i)}, \mathrm{CP}_{g, 1}^{(i i)}, \mathrm{CP}_{g, 2}^{(i i)}$ and $\mathrm{CP}_{g, 3}^{(i i)}$ for the case

$$
c_{l 2}^{(1)}>0, \quad c_{l 3}^{(1)}>0, \quad c_{l 2}^{(1)} \neq c_{l 3}^{(1)} ;
$$

see (92). We have to see if matrices $U_{R}^{(l)}$ and $U_{L}^{(l)}$ of type (a) (see (78) ) or type (b) (see (79) ) can be found such that (91) can be satisfied.

Let us first note that the diagonal form for $C_{l}^{(1)}$ (68) still allows one to redefine $l_{\alpha R}$ and $\left(\nu_{\alpha L}, l_{\alpha L}\right)^{\mathrm{T}}$ for given $\alpha$ by multiplication with an arbitrary phase factor. If $C_{l 23}^{(2)} \neq 0$, we can use this to require, without loss of generality,

$$
C_{l 23}^{(2)}>0
$$

Alternatively, if $C_{l 32}^{(2)} \neq 0$, we can use the above freedom of phase factors to require without loss of generality

$$
C_{l 32}^{(2)}>0 \text {. }
$$

$$
\text { a. The symmetry } C P_{g, 2}^{(i i)}
$$

Now we impose $\mathrm{CP}_{g, 2}^{(i i)}$ symmetry. With $W_{j i}=\delta_{j i}$ (see Tab. (I) we get from (91)

$$
\begin{aligned}
& U_{R}^{(l) \mathrm{T}} C_{l}^{(1) *} U_{L}^{(l) *}=C_{l}^{(1)}, \\
& U_{R}^{(l) \mathrm{T}} C_{l}^{(2) *} U_{L}^{(l) *}=C_{l}^{(2)} .
\end{aligned}
$$

From (68) we have

$$
C_{l}^{(1)}=C_{l}^{(1) *}=C_{l}^{(1) \mathrm{T}} .
$$

Together with (B.4) we get

$$
\left(U_{R}^{(l)} U_{R}^{(l) *}\right)^{\mathrm{T}} C_{l}^{(1)}\left(U_{L}^{(l) *} U_{L}^{(l)}\right)=C_{l}^{(1)} .
$$

From (B.7), (78) and (79) we see that we have only the possibilities

(I) $U_{R}^{(l)}$ and $U_{L}^{(l)}$ both of type (a), or

(II) $U_{R}^{(l)}$ and $U_{L}^{(l)}$ both of type (b).

Consider first (I), then we have the ansatz (see (78))

$$
\begin{aligned}
& U_{R}^{(l)}=e^{i \xi_{R}} \epsilon, \\
& U_{L}^{(l)}=e^{i \xi_{L}} \epsilon,
\end{aligned}
$$

and (B.4) gives

$$
U_{R}^{(l) \mathrm{T}} C_{l}^{(1)}=-C_{l}^{(1)} U_{L}^{(l)} .
$$

Inserting (B.8) in (B.9) gives

$$
\begin{aligned}
& e^{i \xi_{R}} c_{l 2}^{(1)}=e^{i \xi_{L}} c_{l 3}^{(1)}, \\
& e^{i \xi_{R}} c_{l 3}^{(1)}=e^{i \xi_{L}} c_{l 2}^{(1)},
\end{aligned}
$$

which is not possible if (B.1) holds.

Thus we are left with possibility (II), and we can make the ansatz (see (79))

$$
\begin{aligned}
& U_{R}^{(l)}=e^{i \xi_{R}}\left(\begin{array}{cc}
\alpha_{R} & \beta_{R} \\
\beta_{R} & -\alpha_{R}^{*}
\end{array}\right), \\
& U_{L}^{(l)}=e^{i \xi_{L}}\left(\begin{array}{cc}
\alpha_{L} & \beta_{L} \\
\beta_{L} & -\alpha_{L}^{*}
\end{array}\right),
\end{aligned}
$$

where

$$
\begin{aligned}
& \beta_{R} \geq 0, \quad \beta_{L} \geq 0, \\
& \left|\alpha_{R}\right|^{2}+\beta_{R}^{2}=1, \\
& \left|\alpha_{L}\right|^{2}+\beta_{L}^{2}=1 .
\end{aligned}
$$

From (B.4) we get now

$$
U_{R}^{(l) \mathrm{T}} C_{l}^{(1)}=C_{l}^{(1)} U_{L}^{(l)},
$$

which gives

$$
\begin{gathered}
e^{i \xi_{R}}\left(\begin{array}{cc}
\alpha_{R} c_{l 2}^{(1)} & \beta_{R} c_{l 3}^{(1)} \\
\beta_{R} c_{l 2}^{(1)} & -\alpha_{R}^{*} c_{l 3}^{(1)}
\end{array}\right)= \\
e^{i \xi_{L}}\left(\begin{array}{cc}
c_{l 2}^{(1)} \alpha_{L} & c_{l 2}^{(1)} \beta_{L} \\
c_{l 3}^{(1)} \beta_{L} & -c_{l 3}^{(1)} \alpha_{L}^{*}
\end{array}\right) .
\end{gathered}
$$


With (B.1) we see that the equality of the diagonal matrix elements in (B.14) can only be fulfilled if

$$
\left|\alpha_{R}\right|=\left|\alpha_{L}\right| \text {. }
$$

But this implies from (B.12)

$$
\beta_{R}=\beta_{L}
$$

and looking now at the off-diagonal matrix elements in (B.14) we get, due to $c_{l 2}^{(1)} \neq c_{l 3}^{(1)}$,

$$
\beta_{R}=\beta_{L}=0
$$

which implies $\left|\alpha_{R}\right|=\left|\alpha_{L}\right|=1$. Inserting this in (B.11) we see the (B.13) can only be solved if

$$
U_{R}^{(l)}=U_{L}^{(l)}=\left(\begin{array}{cc}
e^{i \xi_{2}} & 0 \\
0 & e^{i \xi_{3}}
\end{array}\right)
$$

Turning now to $C_{l}^{(2)}$ we find from B.5

$$
U_{R}^{(l) \mathrm{T}} C_{l}^{(2) *}=C_{l}^{(2)} U_{L}^{(l)} .
$$

Inserting here (B.18) we find

$$
\begin{aligned}
& \left(\begin{array}{ll}
e^{i \xi_{2}} C_{l 22}^{(2) *} & e^{i \xi_{2}} C_{l 23}^{(2) *} \\
e^{i \xi_{3}} C_{l 32}^{(2) *} & e^{i \xi_{3}} C_{l 33}^{(2) *}
\end{array}\right)= \\
& \left(\begin{array}{cc}
C_{l 22}^{(2)} e^{i \xi_{2}} & C_{l 23}^{(2)} e^{i \xi_{3}} \\
C_{l 32}^{(2)} e^{i \xi_{2}} & C_{l 33}^{(2)} e^{i \xi_{3}}
\end{array}\right)
\end{aligned}
$$

From (B.20) we see immediately that we must have

$$
\begin{aligned}
& C_{l 22}^{(2) *}=C_{l 22}^{(2)}, \\
& C_{l 33}^{(2) *}=C_{l 33}^{(2)} .
\end{aligned}
$$

If $C_{l 23}^{(2)} \neq 0$ we can impose $C_{l 23}^{(2)}>0$; see (B.2). Then (B.20) can only be fulfilled for $e^{i \xi_{2}}=e^{i \xi_{3}}$ and this implies

$$
C_{l 32}^{(2) *}=C_{l 32}^{(2)}
$$

If $C_{l 32}^{(2)} \neq 0$ we can use $(\underline{B .3}$ and come to the conclusion

$$
C_{l 23}^{(2) *}=C_{l 23}^{(2)} .
$$

To summarise: we find as necessary condition for the leptonic Yukawa coupling (90) to allow for a $\mathrm{CP}_{g, 2}^{(i i)}$ symmetry that $C_{l}^{(2)}$ is a real matrix,

$$
C_{l}^{(2) *}=C_{l}^{(2)}
$$

It is easy to see that (B.24) is also sufficient for $\mathrm{CP}_{g, 2}^{(i i)}$ invariance. We just have to set $\xi_{2}=\xi_{3}$ in (B.18) and (B.20).

In the following the $\mathrm{CP}_{g, 2}^{(i i)}$ symmetry condition (B.24) will be supposed to hold.

\section{b. $\quad$ The symmetry $C P_{g, 1}^{(i i)}$}

Now we impose in addition to $\mathrm{CP}_{g, 2}^{(i i)}$ the symmetry $\mathrm{CP}_{g, 1}^{(i i)}$. That is, we look for solutions of (91) with $W_{j i}=$ $\sigma_{j i}^{3}$; see Tab. I. We get then the conditions

$$
\begin{aligned}
U_{R}^{(l) \mathrm{T}} C_{l}^{(1)} U_{L}^{(l) *} & =C_{l}^{(1)}, \\
-U_{R}^{(l) \mathrm{T}} C_{l}^{(2)} U_{L}^{(l) *} & =C_{l}^{(2)},
\end{aligned}
$$

where we already used $C_{l}^{(j)}=C_{l}^{(j) *}, j=1,2$. Note that with (B.6), (B.4) is equivalent to (B.25). Therefore, we can take over all the results from (B.4) up to (B.18). Also here $U_{R}^{(l)}$ and $U_{L}^{(l)}$ must be both of type (a) or both of type (b). Only type (b) is possible and we must have

$$
U_{R}^{(l)}=U_{L}^{(l)}=\left(\begin{array}{cc}
e^{i \xi_{2}} & 0 \\
0 & e^{i \xi_{3}}
\end{array}\right)
$$

From (B.26) we get now

$$
-U_{R}^{(l) \mathrm{T}} C_{l}^{(2)}=C_{l}^{(2)} U_{L}^{(l)},
$$

from which follows that

$$
\begin{gathered}
-\left(\begin{array}{cc}
e^{i \xi_{2}} C_{l 22}^{(2)} & e^{i \xi_{2}} C_{l 23}^{(2)} \\
e^{i \xi_{3}} C_{l 32}^{(2)} & e^{i \xi_{3}} C_{l 33}^{(2)}
\end{array}\right)= \\
\left(\begin{array}{cc}
C_{l 22}^{(2)} e^{i \xi_{2}} & C_{l 23}^{(2)} e^{i \xi_{3}} \\
C_{l 32}^{(2)} e^{i \xi_{2}} & C_{l 33}^{(2)} e^{i \xi_{3}}
\end{array}\right) .
\end{gathered}
$$

This gives immediately as a necessary condition for (B.29) to be solvable

$$
\begin{aligned}
& C_{l 22}^{(2)}=0, \\
& C_{l 33}^{(2)}=0 .
\end{aligned}
$$

Setting

$$
e^{i \xi_{2}}=-e^{i \xi_{3}}
$$

in (B.27) and (B.29), we see that (B.30) is also sufficient for (B.28) and thus (B.25) and (B.26) to have a solution.

The result of this section is that $\mathrm{CP}_{g, 2}^{(i i)}$ and $\mathrm{CP}_{g, 1}^{(i i)}$ together can be implemented as symmetries of $\mathscr{L}_{\mathrm{Yuk}, l}$ if and only if $C_{l}^{(2)}$ is off-diagonal:

$$
C_{l}^{(2)}=\left(\begin{array}{cc}
0 & C_{l 23}^{(2)} \\
C_{l 32}^{(2)} & 0
\end{array}\right)
$$

and real. We shall impose this condition in the following.

$$
\text { c. The symmetry } \mathrm{CP}_{g}^{(i)}
$$

From (91) we get here with $W=\epsilon$ (see Tab. I) and $C_{l}^{(j) *}=C_{l}^{(j)}$

$$
\begin{aligned}
& U_{R}^{(l) \mathrm{T}} C_{l}^{(1)} U_{L}^{(l) *}=C_{l}^{(2)}, \\
& U_{R}^{(l) \mathrm{T}} C_{l}^{(2)} U_{L}^{(l) *}=-C_{l}^{(1)} .
\end{aligned}
$$


From these we get

$$
\left(U_{R}^{(l)} U_{R}^{(l) *}\right)^{\mathrm{T}} C_{l}^{(1)}\left(U_{L}^{(l) *} U_{L}^{(l)}\right)=-C_{l}^{(1)} .
$$

Furthermore, we find from (B.33)

$$
\left|\operatorname{det} C_{l}^{(2)}\right|=\left|\operatorname{det} C_{l}^{(1)}\right|,
$$

from which we get

$$
\left|C_{l 23}^{(2)} C_{l 32}^{(2)}\right|=c_{l 2}^{(1)} c_{l 3}^{(1)} \neq 0 .
$$

Therefore we have $C_{l 23}^{(2)} \neq 0$ and shall use in the following the phase choice $C_{l 23}^{(2)}>0$; see (B.2).

Looking now at (78) and (79) we see that (B.35) can only be satisfied if

(I) $U_{R}^{(l)}$ is of type (a) and $U_{L}^{(l)}$ is of type (b), or

(II) $U_{R}^{(l)}$ is of type (b) and $U_{L}^{(l)}$ is of type (a).

We start with case (I), where we have to set

$$
\begin{aligned}
& U_{R}^{(l)}=e^{i \xi_{R}}\left(\begin{array}{cc}
0 & 1 \\
-1 & 0
\end{array}\right), \\
& U_{L}^{(l)}=e^{i \xi_{L}}\left(\begin{array}{cc}
\alpha & \beta \\
\beta & -\alpha^{*}
\end{array}\right), \quad \beta \geq 0,|\alpha|^{2}+\beta^{2}=1 .
\end{aligned}
$$

From (B.33) and (B.34) we find

$$
U_{R}^{(l) \mathrm{T}} C_{l}^{(1)}=C_{l}^{(2)} U_{L}^{(l)},
$$

from which follows that

$$
e^{i \xi_{R}}\left(\begin{array}{cc}
0 & -c_{l 3}^{(1)} \\
c_{l 2}^{(1)} & 0
\end{array}\right)=e^{i \xi_{L}}\left(\begin{array}{cc}
C_{l 23}^{(2)} \beta & -C_{l 23}^{(2)} \alpha^{*} \\
C_{l 32}^{(2)} \alpha & C_{l 32}^{(2)} \beta
\end{array}\right) .
$$

From (B.36) and (B.41) we see that we must have

$$
\begin{gathered}
\beta=0, \quad|\alpha|=1, \\
C_{l 23}^{(2)}=e^{i\left(\xi_{R}-\xi_{L}\right)} \alpha c_{l 3}^{(1)}, \\
C_{l 32}^{(2)}=e^{i\left(\xi_{R}-\xi_{L}\right)} \alpha^{*} c_{l 2}^{(1)} .
\end{gathered}
$$

With (B.1) and (B.2) (B.43) implies

$$
e^{i\left(\xi_{R}-\xi_{L}\right)} \alpha=1 .
$$

Since also $C_{l 32}^{(2)}$ must be real; see (B.24), we get from (B.44) that also

$$
e^{i\left(\xi_{R}-\xi_{L}\right)} \alpha^{*}= \pm 1
$$

must hold. There are four solutions to (B.45) and (B.46):

$$
\begin{aligned}
& \alpha=\alpha^{*}=e^{i\left(\xi_{L}-\xi_{R}\right)}=+1, \\
& \alpha=\alpha^{*}=e^{i\left(\xi_{L}-\xi_{R}\right)}=-1, \\
& \alpha=-\alpha^{*}=e^{i\left(\xi_{L}-\xi_{R}\right)}=+i, \\
& \alpha=-\alpha^{*}=e^{i\left(\xi_{L}-\xi_{R}\right)}=-i .
\end{aligned}
$$

The corresponding solutions of (B.41) are as follows. From (B.47a) and (B.47b) we get

$$
\begin{aligned}
C_{l}^{(2)} & =\left(\begin{array}{cc}
0 & c_{l 3}^{(1)} \\
c_{l 2}^{(1)} & 0
\end{array}\right), \\
U_{R}^{(l)} & =e^{i \xi_{R}}\left(\begin{array}{cc}
0 & 1 \\
-1 & 0
\end{array}\right), \\
U_{L}^{(l)} & =e^{i \xi_{R}}\left(\begin{array}{cc}
1 & 0 \\
0 & -1
\end{array}\right) .
\end{aligned}
$$

From (B.47c) and (B.47d $)$ we get

$$
\begin{aligned}
C_{l}^{(2)} & =\left(\begin{array}{cc}
0 & c_{l 3}^{(1)} \\
-c_{l 2}^{(1)} & 0
\end{array}\right), \\
U_{R}^{(l)} & =e^{i \xi_{R}}\left(\begin{array}{cc}
0 & 1 \\
-1 & 0
\end{array}\right), \\
U_{L}^{(l)} & =e^{i \xi_{R}}\left(\begin{array}{cc}
-1 & 0 \\
0 & -1
\end{array}\right) .
\end{aligned}
$$

Turning to case (II) we have the ansatz

$$
U_{R}^{(l)}=e^{i \xi_{R}}\left(\begin{array}{cc}
\alpha & \beta \\
\beta & -\alpha^{*}
\end{array}\right), \quad \beta \geq 0,|\alpha|^{2}+\beta^{2}=1,
$$

$$
U_{L}^{(l)}=e^{i \xi_{L}}\left(\begin{array}{cc}
0 & 1 \\
-1 & 0
\end{array}\right) .
$$

From (B.33) and (B.34) we get

$$
C_{l}^{(1)} U_{L}^{(l)}=U_{R}^{(l) \mathrm{T}} C_{l}^{(2)},
$$

from which we find

$$
e^{i \xi_{L}}\left(\begin{array}{cc}
0 & c_{l 2}^{(1)} \\
-c_{l 3}^{(1)} & 0
\end{array}\right)=e^{i \xi_{R}}\left(\begin{array}{cc}
\beta C_{l 32}^{(2)} & \alpha C_{l 23}^{(2)} \\
-\alpha^{*} C_{l 32}^{(2)} & \beta C_{l 23}^{(2)}
\end{array}\right) .
$$

Using (B.36) and (B.2) we find from (B.53)

$$
\begin{gathered}
\beta=0, \quad|\alpha|=1 ; \\
C_{l 23}^{(2)}=e^{i\left(\xi_{L}-\xi_{R}\right)} \alpha^{*} c_{l 2}^{(1)}, \\
C_{l 32}^{(2)}=e^{i\left(\xi_{L}-\xi_{R}\right)} \alpha c_{l 3}^{(1)} .
\end{gathered}
$$

Complex conjugation in (B.55) gives

$$
\begin{aligned}
& C_{l 23}^{(2)}=e^{i\left(\xi_{R}-\xi_{L}\right)} \alpha c_{l 2}^{(1)}, \\
& C_{l 32}^{(2)}=e^{i\left(\xi_{R}-\xi_{L}\right)} \alpha^{*} c_{l 3}^{(1)} .
\end{aligned}
$$

Apart from the exchange of $c_{l 2}^{(1)}$ and $c_{l 3}^{(1)}$ (B.56) is identical to (B.43) and (B.44). Thus we find that (B.56) only has solutions if $\alpha$ and $\exp \left[i\left(\xi_{L}-\xi_{R}\right)\right]$ are equal to one of the four cases shown in (B.47). 
From (B.47a and (B.47b we were

$$
\begin{aligned}
& C_{l}^{(2)}=\left(\begin{array}{cc}
0 & c_{l 2}^{(1)} \\
c_{l 3}^{(1)} & 0
\end{array}\right), \\
& U_{R}^{(l)}=e^{i \xi_{L}}\left(\begin{array}{cc}
1 & 0 \\
0 & -1
\end{array}\right), \\
& U_{L}^{(l)}=e^{i \xi_{L}}\left(\begin{array}{cc}
0 & 1 \\
-1 & 0
\end{array}\right) .
\end{aligned}
$$

From (B.47c) and (B.47d $)$ we get here

$$
\begin{aligned}
& C_{l}^{(2)}=\left(\begin{array}{cc}
0 & c_{l 2}^{(1)} \\
-c_{l 3}^{(1)} & 0
\end{array}\right), \\
& U_{R}^{(l)}=e^{i \xi_{L}}\left(\begin{array}{ll}
1 & 0 \\
0 & 1
\end{array}\right), \\
& U_{L}^{(l)}=e^{i \xi_{L}}\left(\begin{array}{cc}
0 & 1 \\
-1 & 0
\end{array}\right) .
\end{aligned}
$$

We see that requiring the symmetries $\mathrm{CP}_{g, 2}^{(i i)}, \mathrm{CP}_{g, 1}^{(i i)}$ and $\mathrm{CP}_{g}^{(i)}$ to hold leads to only four distinct possibilities for $C_{l}^{(2)}$ as shown in (B.48), (B.49), (B.57) and (B.58) and Tab. III. Of course, all this applies only under the condition that (92) holds. It remains to be seen if these four cases also allow for the implementation of the $\mathrm{CP}_{g, 3}^{(i i)}$ symmetry.

\section{d. The symmetry $C P_{g, 3}^{(i i)}$}

Here we have to look for solutions of (91) setting $W_{j i}=$ $\sigma_{j i}^{1}$; see Tab. I] This gives with $C_{l}^{(j) *}=C_{l}^{(j)}$

$$
\begin{aligned}
& U_{R}^{(l) \mathrm{T}} C_{l}^{(1)} U_{L}^{(l) *}=C_{l}^{(2)}, \\
& U_{R}^{(l) \mathrm{T}} C_{l}^{(2)} U_{L}^{(l) *}=C_{l}^{(1)} .
\end{aligned}
$$

From this we get

$$
\left(U_{R}^{(l)} U_{R}^{(l) *}\right)^{\mathrm{T}} C_{l}^{(1)}\left(U_{L}^{(l) *} U_{L}^{(l)}\right)=C_{l}^{(1)},
$$

which shows that both, $U_{R}^{(l)}$ and $U_{L}^{(l)}$, have to be of type (a) as in (78) or of type (b) as in (79). We have already found that $C_{l}^{(2)}$ can be only as in (B.48), (B.49), (B.57) or (B.58). It is easy to see that none of these cases allows for a solution of B.59) if $U_{R}^{(l)}$ and $U_{L}^{(l)}$ are of type (a). If both $U_{R}^{(l)}$ and $U_{L}^{(l)}$ are of type (b) we have solutions in all four cases. For $C_{l}^{(2)}$ as in (B.48) we get a solution of (B.59) setting

$$
\begin{aligned}
& U_{R}^{(l)}=\sigma^{1}, \\
& U_{L}^{(l)}=\mathbb{1}_{2} .
\end{aligned}
$$

For $C_{l}^{(2)}$ as in (B.49) we set

$$
\begin{aligned}
& U_{R}^{(l)}=\sigma^{1}, \\
& U_{L}^{(l)}=-\sigma^{3} .
\end{aligned}
$$

For $C_{l}^{(2)}$ as in (B.57) we set

$$
\begin{aligned}
& U_{R}^{(l)}=\mathbb{1}_{2}, \\
& U_{L}^{(l)}=\sigma^{1} .
\end{aligned}
$$

For $C_{l}^{(2)}$ as in (B.58) we set

$$
\begin{aligned}
& U_{R}^{(l)}=\sigma^{3}, \\
& U_{L}^{(l)}=\sigma^{1} .
\end{aligned}
$$

This completes the proof that for all cases of $C_{l}^{(2)}$ listed in Tab. II we can implement all symmetries $\mathrm{CP}_{g, 1}^{(i i)}$, $\mathrm{CP}_{g, 2}^{(i i)}, \mathrm{CP}_{g, 3}^{(i i)}$ and $\mathrm{CP}_{g}^{(i)}$. As we have shown, no other form of $C_{l}^{(2)}$ allows this to happen if (92) holds for $C_{l}^{(1)}$.

\section{The equal mass case}

Here we suppose

$$
c_{l 2}^{(1)}=c_{l 3}^{(1)}>0
$$

to hold. We have then

$$
C_{l}^{(1)}=c_{l 2}^{(1)} \mathbb{1}_{2}
$$

in (90). We want to find the general structure of $C_{l}^{(2)}$ compatible with invariance under all four CP transformations.

We start with the $\mathrm{CP}_{g}^{(i)}$ symmetry which requires (91) to hold with $W_{j i}=\epsilon_{j i}$; see Tab. I. This leads to

$$
\begin{gathered}
c_{l 2}^{(1)} U_{R}^{(l) \mathrm{T}} U_{L}^{(l) *}=C_{l}^{(2)}, \\
U_{R}^{(l) \mathrm{T}} C_{l}^{(2) *} U_{L}^{(l) *}=-c_{l 2}^{(1)} \mathbb{1}_{2} .
\end{gathered}
$$

From (B.67) we see that $C_{l}^{(2)} / c_{l 2}^{(1)}$ is a unitary matrix. Therefore we can make a basis change of $l_{\alpha R}$ and $\left(\nu_{\alpha L}, l_{\alpha L}\right)^{\mathrm{T}}$ in order to diagonalise $C_{l}^{(2)}$. Indeed, consider the basis change

$$
\begin{gathered}
l_{\alpha R} \rightarrow V_{\alpha \beta} l_{\beta R}, \\
\left(\begin{array}{c}
\nu_{\alpha L} \\
l_{\alpha L}
\end{array}\right) \rightarrow V_{\alpha \beta}\left(\begin{array}{c}
\nu_{\beta L} \\
l_{\beta L}
\end{array}\right),
\end{gathered}
$$

with $V \in U(2)$ :

$$
V=\left(V_{\alpha \beta}\right), \quad V V^{\dagger}=V^{\dagger} V=\mathbb{1}_{2} .
$$

This leads to

$$
C_{l}^{(j)} \rightarrow V^{\dagger} C_{l}^{(j)} V, \quad j=1,2 .
$$


With a suitable $V$ we can achieve

$$
C_{l}^{(2)}=c_{l 2}^{(1)}\left(\begin{array}{cc}
e^{i \eta_{2}} & 0 \\
0 & e^{i \eta_{3}}
\end{array}\right)
$$

with $\eta_{2}$ and $\eta_{3}$ real. Note that the transformation (B.71) does not affect $C_{l}^{(1)}$ of (B.66).

Taking the complex conjugate of (B.68) and inserting $C_{l}^{(2)}$ from (B.67) we get

$$
\left(U_{R}^{(l)} U_{R}^{(l) *}\right)^{\mathrm{T}}\left(U_{L}^{(l) *} U_{L}^{(l)}\right)=-\mathbb{1}_{2} .
$$

This shows that there are only two possibilities:

(I) $U_{R}^{(l)}$ of type (a); see (78), and $U_{L}^{(l)}$ of type (b), see (79), or

(II) $U_{R}^{(l)}$ of type (b) and $U_{L}^{(l)}$ of type (a).

We start by considering case (I) where we have the ansatz

$$
\begin{aligned}
U_{R}^{(l)} & =e^{i \xi_{R}}\left(\begin{array}{cc}
0 & 1 \\
-1 & 0
\end{array}\right), \\
U_{L}^{(l)} & =e^{i \xi_{L}}\left(\begin{array}{cc}
\alpha & \beta \\
\beta & -\alpha^{*}
\end{array}\right), \quad \beta \geq 0,|\alpha|^{2}+\beta^{2}=1 .
\end{aligned}
$$

From (B.67) and (B.72) we get then

$$
\begin{aligned}
c_{l 2}^{(1)} U_{R}^{(l) \mathrm{T}} & =C_{l}^{(2)} U_{L}^{(l)}, \\
e^{i \xi_{R}}\left(\begin{array}{cc}
0 & -1 \\
1 & 0
\end{array}\right) & =e^{i \xi_{L}}\left(\begin{array}{cc}
e^{i \eta_{2}} \alpha & e^{i \eta_{2}} \beta \\
e^{i \eta_{3}} \beta & -e^{i \eta_{3}} \alpha^{*}
\end{array}\right) .
\end{aligned}
$$

It follows that

$$
\begin{gathered}
\alpha=0, \quad \beta=1, \\
-e^{i \xi_{R}}=e^{i \xi_{L}} e^{i \eta_{2}}, \\
e^{i \xi_{R}}=e^{i \xi_{L}} e^{i \eta_{3}}, \\
\Rightarrow \quad e^{i\left(\xi_{R}-\xi_{L}\right)}=e^{i \eta_{3}}=-e^{i \eta_{2}} .
\end{gathered}
$$

Thus we find here the following solution of (B.67) and (B.68):

$$
\begin{aligned}
C_{l}^{(2)} & =c_{l 2}^{(1)} e^{i \eta_{2}}\left(\begin{array}{cc}
1 & 0 \\
0 & -1
\end{array}\right) \\
& =c_{l 2}^{(1)} e^{i \eta_{2}} \sigma^{3} \\
U_{R}^{(l)} & =e^{i \xi_{R}} \epsilon \\
U_{L}^{(l)} & =-e^{i\left(\xi_{R}-\eta_{2}\right)} \sigma^{1}
\end{aligned}
$$

Turning now to case (II) we have to make the ansatz

$$
\begin{aligned}
& U_{R}^{(l)}=e^{i \xi_{R}}\left(\begin{array}{cc}
\alpha & \beta \\
\beta & -\alpha^{*}
\end{array}\right), \quad \beta \geq 0,|\alpha|^{2}+\beta^{2}=1 \\
& U_{L}^{(l)}=e^{i \xi_{L}} \epsilon .
\end{aligned}
$$

Here we get from (B.67) and (B.68)

$$
c_{l 2}^{(1)} U_{R}^{(l) \mathrm{T}}=-C_{l}^{(2)} U_{L}^{(l)},
$$

from which follows that

$$
e^{i \xi_{R}}\left(\begin{array}{cc}
\alpha & \beta \\
\beta & -\alpha^{*}
\end{array}\right)=e^{i \xi_{L}}\left(\begin{array}{cc}
0 & -e^{i \eta_{2}} \\
e^{i \eta_{3}} & 0
\end{array}\right) .
$$

This can only be fulfilled if

$$
\begin{gathered}
\alpha=0, \quad \beta=1, \\
e^{i\left(\xi_{R}-\xi_{L}\right)}=e^{i \eta_{3}}=-e^{i \eta_{2}} .
\end{gathered}
$$

Inserting this in $\mathrm{B} .72$ we find that $C_{l}^{(2)}$ must again have the form (B.80), and then (B.84) is solved with

$$
\begin{aligned}
& U_{R}^{(l)}=e^{i \xi_{R}} \sigma^{1}, \\
& U_{L}^{(l)}=-e^{i\left(\xi_{R}-\eta_{2}\right)} \epsilon .
\end{aligned}
$$

Thus, $\mathrm{CP}_{g}^{(i)}$ invariance now fixes $C_{l}^{(2)}$ to be of the form (B.80). We shall next impose $\mathrm{CP}_{g, 2}^{(i i)}$ invariance. From (91) we find then with $W_{j i}=\delta_{j i}$ the conditions

$$
\begin{aligned}
U_{R}^{(l) \mathrm{T}} U_{L}^{(l) *} & =\mathbb{1}_{2}, \\
U_{R}^{(l) \mathrm{T}} e^{-i \eta_{2}} \sigma^{3} U_{L}^{(l) *} & =e^{i \eta_{2}} \sigma^{3} .
\end{aligned}
$$

From (B.88) we get immediately

$$
U_{R}^{(l)}=U_{L}^{(l)} .
$$

Inserting this in (B.89) we get

$$
\sigma^{3} U_{L}^{(l)}=e^{-2 i \eta_{2}} U_{L}^{(l)} \sigma^{3} .
$$

We can have two cases.

(I) $U_{L}^{(l)}$ of type (a); see (78),

$$
U_{L}^{(l)}=e^{i \xi_{L}} \epsilon
$$

Inserting this in (B.91) gives

$$
\begin{gathered}
e^{2 i \eta_{2}}=-1 \\
e^{i \eta_{2}}= \pm i
\end{gathered}
$$

(II) $U_{L}^{(l)}$ of type (b); see (79),

$$
U_{L}^{(l)}=e^{i \xi_{L}}\left(\begin{array}{cc}
\alpha & \beta \\
\beta & -\alpha^{*}
\end{array}\right), \quad \beta \geq 0, \quad|\alpha|^{2}+\beta^{2}=1
$$

Now (B.91) gives

$$
\left(\begin{array}{cc}
\alpha & \beta \\
-\beta & \alpha^{*}
\end{array}\right)=e^{-2 i \eta_{2}}\left(\begin{array}{cc}
\alpha & -\beta \\
\beta & \alpha^{*}
\end{array}\right)
$$




\begin{tabular}{|c||c||c|c|}
\hline \multicolumn{1}{|c||}{$C_{l}^{(2)} / c_{l 2}^{(1)}$} & $\mathrm{CP}_{g, 1}^{(i)}$ & \multicolumn{2}{c|}{$\mathrm{CP}_{g, 3}^{(i i)}$} \\
\hline & $U_{R}^{(l)}=U_{L}^{(l)}$ & $U_{R}^{(l)}$ & $U_{L}^{(l)}$ \\
\hline$\sigma^{3}$ & $\epsilon$ & $\sigma^{3}$ & $\mathbb{1}_{2}$ \\
$-\sigma^{3}$ & $\sigma^{1}$ & $-\sigma^{3}$ & $\mathbb{1}_{2}$ \\
$i \sigma^{3}$ & $\mathbb{1}_{2}$ & $i \sigma^{3}$ & $\mathbb{1}_{2}$ \\
$-i \sigma^{3}$ & $\mathbb{1}_{2}$ & $-i \sigma^{3}$ & $\mathbb{1}_{2}$ \\
\hline
\end{tabular}

TABLE VI: Matrices $U_{R}^{(l)}$ and $U_{L}^{(l)}$ solving (91) for the allowed forms of $C_{l}^{(2)}$, (B.101), for the symmetries $\mathrm{CP}_{g, 1}^{(i i)}$ and $\mathrm{CP}_{g, 3}^{(i i)}$.

from which we get

$$
\begin{aligned}
& \left(1+e^{-2 i \eta_{2}}\right) \beta=0, \\
& \left(1-e^{-2 i \eta_{2}}\right) \alpha=0 .
\end{aligned}
$$

The solutions of (B.97) and (B.98) and are as follows. For $\alpha \neq 0$ we get

$$
\begin{gathered}
e^{-2 i \eta_{2}}=1, \\
e^{i \eta_{2}}= \pm 1, \\
\beta=0, \quad|\alpha|=1 .
\end{gathered}
$$

For $\alpha=0$ we must have $\beta \neq 0$; see $(\overline{B .95})$, and we get

$$
\begin{gathered}
e^{-2 i \eta_{2}}=-1, \\
e^{i \eta_{2}}= \pm i, \\
\alpha=0, \quad \beta=1 .
\end{gathered}
$$

In summary: we see from (B.80), (B.94), (B.99) and (B.100) that imposition of the $\mathrm{CP}_{g}^{(i)}$ and $\mathrm{CP}_{g, 2}^{(i i)}$ symmetries requires $C_{l}^{(2)}$ to be of one of the following forms:

$$
\begin{aligned}
& C_{l}^{(2)}=c_{l 2}^{(1)} \sigma^{3}, \\
& C_{l}^{(2)}=-c_{l 2}^{(1)} \sigma^{3}, \\
& C_{l}^{(2)}=i c_{l 2}^{(1)} \sigma^{3}, \\
& C_{l}^{(2)}=-i c_{l 2}^{(1)} \sigma^{3} .
\end{aligned}
$$

For all these cases we can also implement $\mathrm{CP}_{g, 1}^{(i i)}$ and $\mathrm{CP}_{g, 3}^{(i i)}$ invariance transformations. That is, we can always find appropriate $U_{R}^{(l)}$ and $U_{L}^{(l)}$ solving (91) with $W_{j i}=\sigma_{j i}^{3}$ and $W_{j i}=\sigma_{j i}^{1}$ for $\mathrm{CP}_{g, 1}^{(i i)}$ and $\mathrm{CP}_{g, 3}^{(i i)}$, respectively; see Tab. I We list the corresponding matrices in Tab. VI.

\section{The massive plus massless case}

Here we discuss the case that we have one massive and one massless lepton; that is, we suppose

$$
c_{l 2}^{(1)}=0, \quad c_{l 3}^{(1)}>0
$$

in (68). In fact, we shall start here from the more general case

$$
c_{l 2}^{(1)} \geq 0, \quad c_{l 3}^{(1)}>0
$$

and prescribe matrices $U_{R}^{(l)}$ and $U_{L}^{(l)}$ for the leptons for our four CP symmetries as shown in Tab. IV] We require (91) to hold with the matrices of Tab. IV for all four $\mathrm{CP}_{g}$ symmetries.

We start with $\mathrm{CP}_{g, 2}^{(i i)}=\mathrm{CP}_{s}$. From (91) and Tab. IV we get here

$$
\begin{aligned}
& C_{l}^{(1) *}=C_{l}^{(1)}, \\
& C_{l}^{(2) *}=C_{l}^{(2)} .
\end{aligned}
$$

Thus $C_{l}^{(2)}$ is constrained to be a real matrix. Requiring now also $\mathrm{CP}_{g, 1}^{(i i)}$ invariance we get from (91) and Tab. IV

$$
\begin{aligned}
\left(\begin{array}{cc}
-1 & 0 \\
0 & 1
\end{array}\right)\left(\begin{array}{cc}
c_{l 2}^{(1)} & 0 \\
0 & c_{l 3}^{(1)}
\end{array}\right) & =\left(\begin{array}{cc}
c_{l 2}^{(1)} & 0 \\
0 & c_{l 3}^{(1)}
\end{array}\right), \\
\left(\begin{array}{cc}
1 & 0 \\
0 & -1
\end{array}\right)\left(\begin{array}{ll}
C_{l 22}^{(2)} & C_{l 23}^{(2)} \\
C_{l 32}^{(2)} & C_{l 33}^{(2)}
\end{array}\right) & =\left(\begin{array}{cc}
C_{l 22}^{(2)} & C_{l 23}^{(2)} \\
C_{l 32}^{(2)} & C_{l 33}^{(2)}
\end{array}\right) .
\end{aligned}
$$

It follows that

$$
\begin{aligned}
c_{l 2}^{(1)} & =0, \\
C_{l 32}^{(2)} & =C_{l 33}^{(2)}=0 .
\end{aligned}
$$

Thus, a massless lepton $l_{2}$ which is implied by (B.107) is here a consequence of our ansatz for the $\mathrm{CP}_{g, 1}^{(i i)}$ symmetry.

The next symmetry to consider is $\mathrm{CP}_{g}^{(i)}$ where we get from (91) and Tab. IV

$$
\left(\begin{array}{cc}
0 & -1 \\
1 & 0
\end{array}\right)\left(\begin{array}{cc}
0 & 0 \\
0 & c_{l 3}^{(1)}
\end{array}\right)\left(\begin{array}{ll}
0 & 1 \\
1 & 0
\end{array}\right)=C_{l}^{(2)} .
$$

It follows that

$$
C_{l}^{(2)}=\left(\begin{array}{cc}
-c_{l 3}^{(1)} & 0 \\
0 & 0
\end{array}\right)
$$

The remaining relations for the $\mathrm{CP}_{g}^{(i)}$ and $\mathrm{CP}_{g, 3}^{(i i)}$ symmetries following from (91) and Tab. IV are easily seen to hold if (B.107) and (B.110) are true.

Now we give a general discussion of the case one massless and one massive lepton. That is, we suppose (B.102 to hold and impose invariance under $\mathrm{CP}_{g}^{(i)}, \mathrm{CP}_{g, 1}^{(i i)}, \mathrm{CP}_{g, 2}^{(i i)}$ and $\mathrm{CP}_{g, 3}^{(i i)}$.

We start with $\mathrm{CP}_{g}^{(i)}$ where we look for matrices $U_{R}^{(l)}$ and $U_{L}^{(l)}$ such that

$$
\begin{aligned}
& U_{R}^{(l) \mathrm{T}} C_{l}^{(1) *} U_{L}^{(l) *}=C_{l}^{(2)}, \\
& U_{R}^{(l) \mathrm{T}} C_{l}^{(2) *} U_{L}^{(l) *}=-C_{l}^{(1)} .
\end{aligned}
$$


See (91) and Tab. I. From (B.111) we get

$$
\left(U_{R}^{(l) *} U_{R}^{(l)}\right)^{\mathrm{T}} C_{l}^{(1)}\left(U_{L}^{(l)} U_{L}^{(l) *}\right)=-C_{l}^{(1)} .
$$

This shows that we have only two possibilities,

(I) $U_{R}^{(l)}$ of type (a), see (78), and $U_{L}^{(l)}$ of type (b), see (79), or

(II) $U_{R}^{(l)}$ of type (b) and $U_{L}^{(l)}$ of type (a).

For the case (I) we have the ansatz

$$
\begin{aligned}
& U_{R}^{(l)}=e^{i \xi_{R}}\left(\begin{array}{cc}
0 & 1 \\
-1 & 0
\end{array}\right), \\
& U_{L}^{(l)}=e^{i \xi_{L}}\left(\begin{array}{cc}
\alpha & \beta \\
\beta & -\alpha^{*}
\end{array}\right), \quad \beta \geq 0,|\alpha|^{2}+\beta^{2}=1 .
\end{aligned}
$$

From (B.111) we get here

$$
\begin{aligned}
C_{l}^{(2)} & =U_{R}^{(l) \mathrm{T}} C_{l}^{(1) *} U_{L}^{(l) *} \\
& =c_{l 3}^{(1)} e^{i\left(\xi_{R}-\xi_{L}\right)}\left(\begin{array}{cc}
-\beta & \alpha \\
0 & 0
\end{array}\right) .
\end{aligned}
$$

That is, with $C_{l}^{(2)}$ from (B.114 we have $\mathrm{CP}_{g}^{(i)}$ invariance. The phase factor $\exp \left[i\left(\xi_{R}-\xi_{L}\right)\right]$ can be absorbed in the definition of the field $l_{2}(x)$. Thus, the only independent solutions are here

$$
C_{l}^{(2)}=c_{l 3}^{(1)}\left(\begin{array}{cc}
-\beta & \alpha \\
0 & 0
\end{array}\right)
$$

For the case (II) we have the ansatz

$$
\begin{aligned}
U_{R}^{(l)} & =e^{i \xi_{R}}\left(\begin{array}{cc}
\alpha & \beta \\
\beta & -\alpha^{*}
\end{array}\right), \quad \beta \geq 0,|\alpha|^{2}+\beta^{2}=1 \\
U_{L}^{(l)} & =e^{i \xi_{L}}\left(\begin{array}{cc}
0 & 1 \\
-1 & 0
\end{array}\right) .
\end{aligned}
$$

From (B.111) we get here

$$
C_{l}^{(2)}=c_{l 3}^{(1)} e^{i\left(\xi_{R}-\xi_{L}\right)}\left(\begin{array}{cc}
-\beta & 0 \\
\alpha^{*} & 0
\end{array}\right)
$$

Absorbing the phase factor $\exp \left[i\left(\xi_{R}-\xi_{L}\right)\right]$ in the definition of the doublet fields $\left(\nu_{2} L(x), l_{2} L(x)\right)^{\mathrm{T}}$ we get here the independent solutions as follows:

$$
C_{l}^{(2)}=c_{l 3}^{(1)}\left(\begin{array}{cc}
-\beta & 0 \\
\alpha^{*} & 0
\end{array}\right)
$$

Thus we find that $\mathrm{CP}_{g}^{(i)}$ symmetry requires $C_{l}^{(2)}$ to be of the form (B.115) or (B.118). Turning now to the symmetries $\mathrm{CP}_{g, 1}^{(i i)}, \mathrm{CP}_{g, 2}^{(i i)}$ and $\mathrm{CP}_{g, 3}^{(i i)}$ we find that they can always be implemented for $C_{l}^{(2)}$ of (B.115 or (B.118). Thus, these symmetries give no further restrictions for $C_{l}^{(2)}$. However, if we require the absence of FCNCs we must have $\alpha=0$ in (B.115) and (B.118). Then we have $\beta=1$, and we are led to the unique form

$$
C_{l}^{(2)}=c_{l 3}^{(1)}\left(\begin{array}{cc}
-1 & 0 \\
0 & 0
\end{array}\right)
$$

entering in the coupling (102).

\section{APPENDIX C: INVARIANT COUPLINGS FOR TWO QUARK FAMILIES, DETAILS}

Here we study the Yukawa coupling $\mathscr{L}_{\text {Yuk }, q}$ (110) supposing

$$
c_{d 2}^{(1)} \geq 0, \quad c_{d 3}^{(1)}>0
$$

and prescribing the matrices $U_{R}^{(d)}$ and $U_{L}^{(u)}$ for the $\mathrm{CP}_{g}$ transformations of the quark fields (see (173) ) as follows:

$$
\begin{aligned}
& U_{R}^{(d)}=U_{R}^{(l)}, \\
& U_{L}^{(u)}=U_{L}^{(l)} .
\end{aligned}
$$

Here $U_{R}^{(l)}$ and $U_{L}^{(l)}$ are as in Tab. IV Note that the ansatz (C.2) refers to the $d_{\alpha R}^{\prime}$ fields and (C.3) to the fields $\left(u_{\alpha L}, d_{\alpha L}^{\prime}\right)^{\mathrm{T}}$.

Without loss of generality we may suppose $C_{q}^{(1)}$ to be of the form (70) and (71). Imposing now $\mathrm{CP}_{g, 1}^{(i i)}$ invariance we find from (86) with $W_{j i}=\sigma_{j i}^{3}$ (see Tab. I)

$$
U_{R}^{(d) \mathrm{T}} C_{q}^{(j) *} U_{L}^{(u) *} \sigma_{j i}^{3}=C_{q}^{(i)}
$$

Inserting here $U_{R}^{(d)}$ and $U_{L}^{(u)}$ according to (C.2), (C.3) and Tab. IV we get

$$
\left(-\sigma^{3}\right) C_{q}^{(1)}=C_{q}^{(1)},
$$

from which follows that

$$
-\sigma^{3} V\left(\begin{array}{cc}
c_{d 2}^{(1)} & 0 \\
0 & c_{d 3}^{(1)}
\end{array}\right) V^{\dagger}=V\left(\begin{array}{cc}
c_{d 2}^{(1)} & 0 \\
0 & c_{d 3}^{(1)}
\end{array}\right) V^{\dagger}
$$

and

$$
\left(\begin{array}{cc}
-V_{22} c_{d 2}^{(1)} & -V_{23} c_{d 3}^{(1)} \\
V_{32} c_{d 2}^{(1)} & V_{33} c_{d 3}^{(1)}
\end{array}\right)=\left(\begin{array}{ccc}
V_{22} c_{d 2}^{(1)} & V_{23} c_{d 3}^{(1)} \\
V_{32} c_{d 2}^{(1)} & V_{33} c_{d 3}^{(1)}
\end{array}\right)
$$

With (C.1) we get

$$
V_{23}=0,
$$

which implies from (71)

$$
V_{22}=V_{33}=1 .
$$


Inserting this in (C.7) gives

$$
c_{d 2}^{(1)}=0
$$

Thus our symmetry requires a massless $d_{2}$-quark

$$
m_{d 2}=c_{d 2}^{(1)} \frac{v_{0}}{\sqrt{2}}=0 .
$$

The analysis of the remaining symmetry requirements runs now along exactly the same lines as for the leptons in section $\mathrm{B}$. The result is that our principle of maximal CP symmetry with the ansatz (C.2) and (C.3) for the matrices $U_{R}^{(d)}$ and $U_{L}^{(u)}$ requires

$$
\begin{aligned}
C_{q}^{(1)} & =\left(\begin{array}{cc}
0 & 0 \\
0 & c_{d 3}^{(1)}
\end{array}\right), \\
C_{q}^{(2)} & =\left(\begin{array}{cc}
-c_{d 3}^{(1)} & 0 \\
0 & 0
\end{array}\right) .
\end{aligned}
$$

Finally, we make some remarks on the general analysis for the case of one massless quark pair $\left(u_{2}, d_{2}\right)$ and one massive pair $\left(u_{3}, d_{3}\right)$. That is, we suppose

$$
\begin{aligned}
c_{u 2}^{(1)} & =0, & & c_{u 3}^{(1)}>0, \\
c_{d 2}^{(1)} & =0, & & c_{d 3}^{(1)}>0 .
\end{aligned}
$$

As for the lepton sector, we can show that the principle of maximal $\mathrm{CP}$ invariance together with the requirement of absence of FCNCs leads to the following structure of the quark-Higgs coupling matrices (see (B.119) ):

$$
\begin{aligned}
C_{q}^{(1)} & =\left(\begin{array}{cc}
0 & 0 \\
0 & c_{u 3}^{(1)}
\end{array}\right), \\
C_{q}^{\prime(2)} & =\left(\begin{array}{cc}
-c_{u 3}^{(1)} & 0 \\
0 & 0
\end{array}\right), \\
\tilde{C}_{q}^{(1)} & =\left(\begin{array}{cc}
0 & 0 \\
0 & c_{d 3}^{(1)}
\end{array}\right), \\
\tilde{C}_{q}^{(2)} & =\left(\begin{array}{cc}
-c_{d 3}^{(1)} & 0 \\
0 & 0
\end{array}\right) .
\end{aligned}
$$

Here $\tilde{C}_{q}^{(j)}$ are the CKM rotated matrices according to (113). From the discussion of the lepton case (see (B.111) ff) we see that $\mathrm{CP}_{g}^{(i)}$ invariance is implementable for (C.14) and (C.15) only with certain matrices $U_{R}^{(u)}$, $U_{L}^{(u)}$ in (73) and certain CKM rotated matrices $\tilde{U}_{R}^{(d)}$, $\tilde{U}_{L}^{(u)}$ for the CKM rotated fields in (111). Here we have according to 82

$$
\begin{aligned}
& \tilde{U}_{R}^{(d)}=V^{\dagger} U_{R}^{(d)} V^{*}, \\
& \tilde{U}_{L}^{(u)}=V^{\dagger} U_{L}^{(u)} V^{*} .
\end{aligned}
$$

We have for $U_{R}^{(u)}, U_{L}^{(u)}$ and $\tilde{U}_{R}^{(d)}, \tilde{U}_{L}^{(u)}$ only the possibilities (I) and (II) of (B.113) and (B.116), respectively, with $\alpha=0$ and $\xi_{R}=\xi_{L}$. We have to check for the resulting four cases if we can then fulfil (C.17) or, equivalently, with $V=V^{*}($ see (71) $)$

$$
V \tilde{U}_{L}^{(u)}=U_{L}^{(u)} V .
$$

For both, $U_{R}^{(u)}, U_{L}^{(u)}$ and $\tilde{U}_{R}^{(d)}, \tilde{U}_{L}^{(u)}$, of the type (B.113) with $\alpha=0$ and $\xi_{R}=\xi_{L}$ we have

$$
\begin{aligned}
& U_{R}^{(u)}=e^{i \xi} \epsilon, \\
& U_{L}^{(u)}=e^{i \xi} \sigma^{1}, \\
& \tilde{U}_{R}^{(d)}=e^{i \tilde{\xi}} \epsilon, \\
& \tilde{U}_{L}^{(d)}=e^{i \tilde{\xi}} \sigma^{1} .
\end{aligned}
$$

Then (C.18) can only be fulfilled in two cases. The first solution is

$$
\begin{gathered}
\vartheta=0, \\
e^{i \tilde{\xi}}=e^{i \xi},
\end{gathered}
$$

implying

$$
V=\mathbb{1}_{2}, \quad U_{R}^{(d)}=U_{R}^{(u)} .
$$

The second solution is

$$
\begin{gathered}
\vartheta=\pi / 2, \\
e^{i \tilde{\xi}}=-e^{i \xi},
\end{gathered}
$$

implying

$$
V=\epsilon, \quad U_{R}^{(d)}=-U_{R}^{(u)} .
$$

For both, $U_{R}^{(u)}, U_{L}^{(u)}$ and $\tilde{U}_{R}^{(d)}, \tilde{U}_{L}^{(u)}$, of the type (B.116) with $\alpha=0$ and $\xi_{R}=\xi_{L}$ we have

$$
\begin{aligned}
& U_{R}^{(u)}=e^{i \xi} \sigma^{1}, \\
& U_{L}^{(u)}=e^{i \xi} \epsilon, \\
& \tilde{U}_{R}^{(d)}=e^{i \tilde{\xi}} \sigma^{1}, \\
& \tilde{U}_{L}^{(u)}=e^{i \tilde{\xi}} \epsilon .
\end{aligned}
$$

Inserting this in C.18 we find

$$
e^{i \tilde{\xi}}=e^{i \xi}
$$

but no restriction on $\vartheta$. We get then from (C.16)

$$
U_{R}^{(d)}=e^{i \xi}\left(\begin{array}{cc}
\sin 2 \vartheta & \cos 2 \vartheta \\
\cos 2 \vartheta & -\sin 2 \vartheta
\end{array}\right)
$$

For the remaining cases, $U_{R}^{(u)}, U_{L}^{(u)}$ according to (B.113) and $\tilde{U}_{R}^{(d)}, \tilde{U}_{L}^{(u)}$ according to (B.116), or vice versa, there is no solution of (C.18) possible. 
Thus we see that, strictly speaking, the principle of maximal $\mathrm{CP}$ invariance plus absence of FCNCs gives no restriction on the angle $\vartheta$ in the $2-3$ sector of the CKM matrix. But perhaps we can argue that also the righthanded quarks $u_{\alpha R}, d_{\alpha R}^{\prime}$ should belong to some multiplet of a bigger gauge group as would be possible in grand unified scenarios. Then a natural requirement could be $U_{R}^{(u)}=U_{R}^{(d)}$. From (C.21) and (C.23) as well as (C.24) and (C.26) we see that we have then only the solution $\vartheta=0$ leading to $V=\mathbb{1}_{2}$.

\section{APPENDIX D: CP $_{g}$ INVARIANCES AND CONVENTIONAL DISCRETE SYMMETRIES}

In section 5 we have presented our final result for the Yukawa coupling term being compatible with the principle of maximal CP invariance; see (120). There we have also summarised the transformations corresponding to the invariances $\mathrm{CP}_{g}^{(i)}$ to $\mathrm{CP}_{g, 3}^{(i i)}$ see Tab. $\mathrm{V}$ We discuss now briefly the relation of these results to conventional discrete symmetries.

Instead of the $\mathrm{CP}_{g}^{(i)}, \mathrm{CP}_{g, 1}^{(i i)}, \mathrm{CP}_{g, 2}^{(i i)}$ and $\mathrm{CP}_{g, 3}^{(i i)}$ invariances we can also consider the standard $\mathrm{CP}$ transformation $\mathrm{CP}_{g, 2}^{(i i)} \equiv \mathrm{CP}_{s}$ and the transformations

$$
\begin{aligned}
\mathrm{D}^{(i)} & \equiv \mathrm{CP}_{g}^{(i)} \circ \mathrm{CP}_{s}, \\
\mathrm{D}_{1}^{(i i)} & \equiv \mathrm{CP}_{g, 1}^{(i i)} \circ \mathrm{CP}_{s}, \\
\mathrm{D}_{3}^{(i i)} & \equiv \mathrm{CP}_{g, 3}^{(i i)} \circ \mathrm{CP}_{s},
\end{aligned}
$$

The transformations (D.1) imply for the first generation fermions from (61) a sign change

$$
\begin{aligned}
\left(\begin{array}{c}
\nu_{1 L}(x) \\
l_{1 L}(x)
\end{array}\right) & \rightarrow-\left(\begin{array}{l}
\nu_{1 L}(x) \\
l_{1 L}(x)
\end{array}\right), \\
l_{1 R}(x) & \rightarrow-l_{1 R}(x), \\
\left(\begin{array}{c}
u_{1 L}(x) \\
d_{1 L}(x)
\end{array}\right) & \rightarrow-\left(\begin{array}{l}
u_{1 L}(x) \\
d_{1 L}(x)
\end{array}\right), \\
u_{1 R}(x) & \rightarrow-u_{1 R}(x), \\
d_{1 R}(x) & \rightarrow-d_{1 R}(x) .
\end{aligned}
$$

For the Higgs fields we get, generically,

$$
\varphi_{i}(x) \rightarrow \widetilde{\widetilde{W}}_{i j} \varphi_{j}(x)
$$

\begin{tabular}{|c|c|c|c|}
\hline $\mathrm{D}$ & $\widetilde{\widetilde{W}}$ & $\widetilde{\widetilde{U}}_{R}^{(l)}=\widetilde{\widetilde{U}}_{R}^{(u)}=\widetilde{\widetilde{U}}_{R}^{(d)}$ & $\widetilde{\widetilde{U}}_{L}^{(l)}=\widetilde{\widetilde{U}}_{L}^{(u)}$ \\
\hline \hline $\mathrm{D}^{(i)}$ & $\epsilon$ & $-\epsilon$ & $-\sigma^{1}$ \\
$\mathrm{D}_{1}^{(i i)}$ & $\sigma^{3}$ & $\sigma^{3}$ & $-\mathbb{1}_{2}$ \\
$\mathrm{D}_{3}^{(i i)}$ & $\sigma^{1}$ & $\sigma^{1}$ & $-\sigma^{1}$ \\
\hline
\end{tabular}

TABLE VII: The matrices for the transformations of the Higgs fields and of the second and the third generation fermions under the discrete symmetries $\mathrm{D}^{(i)}, \mathrm{D}_{2}^{(i i)}$, and $\mathrm{D}_{3}^{(i i)}$.

and for the second and third fermion families

$$
\begin{aligned}
\left(\begin{array}{c}
\nu_{\alpha L}(x) \\
l_{\alpha L}(x)
\end{array}\right) & \rightarrow \widetilde{\widetilde{U}}_{L \alpha \beta}^{(l)}\left(\begin{array}{c}
\nu_{\beta L}(x) \\
l_{\beta L}(x)
\end{array}\right), \\
l_{\alpha R}(x) & \rightarrow \widetilde{\widetilde{U}}_{R \alpha \beta}^{(l)} l_{\beta R}(x), \\
\left(\begin{array}{c}
u_{\alpha L}(x) \\
d_{\alpha L}(x)
\end{array}\right) & \rightarrow \widetilde{\widetilde{U}}_{L \alpha \beta}^{(u)}\left(\begin{array}{c}
u_{\beta L}(x) \\
d_{\beta L}(x)
\end{array}\right), \\
u_{\alpha R}(x) & \rightarrow \widetilde{\widetilde{U}}_{R \alpha \beta}^{(u)} u_{\beta R}(x), \\
d_{\alpha R}(x) & \rightarrow \widetilde{\widetilde{U}}_{R \alpha \beta}^{(d)} d_{\beta R}(x),
\end{aligned}
$$

$\alpha, \beta \in\{2,3\}$. Here the matrices $\widetilde{\widetilde{W}}$ and $\widetilde{\widetilde{U}}$ are given in Tab. VII

Clearly, imposing the invariances $\mathrm{CP}_{g}^{(i)}$ to $\mathrm{CP}_{g, 3}^{(i i)}$ with the transformations specified in Tab. $\mathrm{V}$ is equivalent to imposing $\mathrm{CP}_{s}$ plus the discrete invariances $\mathrm{D}^{(i)}$ to $\mathrm{D}_{3}^{(i i)}$ as specified in Tab. VII But in our opinion the latter procedure would be completely ad hoc. We could give no physical argument for considering just the specific transformations shown above for the Higgs fields, the first, the second and the third fermion families. Why would we group the second and third families together? On the other hand, we hope to have shown in the main text of this paper that the principle of maximal $\mathrm{CP}$ invariance provides us with a clear physical argument to single out the form of the Higgs potential (27) and of the Yukawa coupling term (120).
[1] S. Weinberg, Phys. Rev. Lett. 19 (1967) 1264.

[2] A. Salam, Originally printed in *Svartholm: Elementary Particle Theory, Proceedings Of The Nobel Symposium Held 1968 At Lerum, Sweden*, Stockholm 1968, 367-377

[3] S. L. Glashow, J. Iliopoulos and L. Maiani, Phys. Rev. D 2 (1970) 1285

[4] O. Nachtmann, "Elementary Particle Physics: Concepts
And Phenomena", Springer, Berlin (1990).

[5] J. F. Gunion and H. E. Haber, Phys. Rev. D 72 (2005) 095002 hep-ph/0506227v2].

[6] C. C. Nishi, Phys. Rev. D $74 \quad$ (2006) 036003 hep-ph/0605153.

[7] I. F. Ginzburg and M. Krawczyk, Phys. Rev. D 72 (2005) 115013 hep-ph/0408011. 
[8] I. P. Ivanov, Phys. Rev. D $\mathbf{7 5}$ (2007) 035001 hep-ph/0609018.

[9] I. P. Ivanov, arXiv:0710.3490 [hep-ph].

[10] M. Kobayashi and T. Maskawa, Prog. Theor. Phys. 49 (1973) 652

[11] A. Barroso, P. M. Ferreira and R. Santos, Phys. Lett. B 652 (2007) 181 hep-ph/0702098.

[12] J. M. Gerard and M. Herquet, Phys. Rev. Lett. 98 (2007) 251802 hep-ph/0703051.

[13] R. Barbieri and L. J. Hall, hep-ph/0510243.

[14] L. Fromme, S. J. Huber and M. Seniuch, JHEP 0611 (2006) 038 hep-ph/0605242.

[15] G. C. Branco, M. N. Rebelo and J. I. Silva-Marcos, Phys. Lett. B 614 (2005) 187 hep-ph/0502118.
[16] M. Maniatis, A. von Manteuffel, O. Nachtmann and F. Nagel, Eur. Phys. J. C 48 (2006) 805 hep-ph/0605184.

[17] M. Maniatis, A. von Manteuffel and O. Nachtmann, Eur. Phys. J. C (in press), DOI: 10.1140/epjc/s10052-0080712-5 (2008), arXiv:0707.3344 [hep-ph].

[18] W. M. Yao et al. [Particle Data Group], J. Phys. G 33, (2006) 1.

[19] M. Jamin, J. A. Oller and A. Pich, Phys. Rev. D 74 (2006) 074009 hep-ph/0605095.

[20] N. Brambilla et al. [Quarkonium Working Group], CERN-2005-005, Geneva (2005), hep-ph/0412158. 